\title{
Logística y optimización del servicio de recogida de residuos urbanos en áreas rurales
}

\author{
J. M. Fernández González \\ Director Técnico, IDEUS, España \\ J. García Pérez \\ Jefe Departamento Ingeniería industrial, IDEUS, España \\ A. Ruiz Lozano \\ Consultor, IDEUS, España \\ D. Villaplana Cerdá \\ Consultor, IDEUS, España
}

\section{RESUMEN}

La Ley 7/1985, de 2 de abril, reguladora de las Bases de Régimen Local, establece que en todos los municipios de España, las administraciones locales deben prestar, entre otros, el servicio de recogida y transporte de residuos urbanos (RU).

En España, con una población total de 45.000.000 habitantes, y un ratio de generación de RU de 1,4 Kg/hab/día, se generan diariamente 63.000 Tn de RU que son transportados hasta plantas de transferencia o plantas de tratamiento y eliminación.

La mayor parte de estos municipios, el $83,8 \%$, tienen menos de 5.000 habitantes y un volumen de generación de residuos diario muy por debajo de la capacidad de carga de los camiones recolectores, por lo que no se consigue un transporte eficiente de los mismos.

La aplicación de nuevas tecnologías así como la mancomunación para la prestación del servicio de recogida de RU consigue un transporte mucho más eficaz desde el punto de vista económico y ambiental.

Entre estas tecnologías se diferencian las que se aplican a los camiones recolectores: dispositivos de control de flotas, sistemas de pesaje en chasis o pesaje embarcado, compartimentación de camiones; y los que se aplican a los contenedores receptores de los RU: sensores de llenado y sensores de manipulación.

La presente investigación pretende cuantificar los ahorros económicos y ambientales que pueden obtenerse en los servicios de recogida de RU mediante la aplicación de nuevas tecnologías y la mancomunación de los mismos.

Con la finalidad de validar esos resultados, se comprueban los obtenidos tras la implantación de estas medidas en el Consorcio para el Desarrollo de la Vega Sierra Elvira, compuesto por un conjunto de municipios del cinturón metropolitano de Granada, para finalizar determinando los potenciales ahorros en un grupo de municipios de la Vega Baja de la Provincia de Alicante, de aplicarse similares actuaciones.

Para el primer territorio estudiado, la implementación de la recogida conjunta para 14 municipios con una población de 66.000 habitantes, ha supuesto un ahorro medio de 13,15 $€ /$ Tn y una reducción de emisiones de GEI de $1,71 \mathrm{KgCO}_{2 \text { eq }} / \mathrm{Tn}$ de RU. En el segundo territorio puede suponer un ahorro medio de 5,17 €/Tn y la reducción de 2,09 $\mathrm{KgCO}_{2 \text { eq }} / \mathrm{Tn}$ en GEI. 


\section{INTRODUCCIÓN}

Se define recogida y transporte de residuos como la recolección de los mismos por el personal y el equipo disponible para ello, con la finalidad de ser trasladados hasta el lugar de tratamiento o eliminación, o bien hasta una planta de transferencia intermedia (Hontoria y Zamorano, 2000).

Según el artículo 26.1 de la Ley 7/1985, de 2 de abril, Reguladoras de las Bases de Régimen Local y el artículo 12.5 de la Ley 22/2011, de 28 de julio, de Residuos y Suelos Contaminados, la recogida y el transporte de residuos es un servicio que deben prestarlo obligatoriamente los municipios, que regulan mediante las ordenanzas municipales.

España está caracterizada por el elevado número de municipios con bajos niveles de población; concretamente existen 8.092 municipios, de los que el 86\% tienen menos de 5.000 habitantes y el $32 \%$ entre 250 y 1.000 ; esta situación dificulta la gestión de los residuos para los municipios, por diferentes razones ambientales, económicas y de escala, afectando a la calidad del servicio prestado (Zamorano et al., 2015).

Las emisiones de gases de efecto invernadero (GEI) procedentes del transporte en España crecieron un 56,8\% en el periodo 1990-2011 (Prieto et al., 2014). Las emisiones de $\mathrm{CO}_{2 \mathrm{eq}}$ debidas al transporte de los vehículos de carga en el año 2012 alcanzaron el 19,46\% del total de las emisiones debidas al transporte (mercancías y personas, y viario, marítimo y ferroviario) que suponen el $40 \%$ del total de emisiones de gases de efecto invernadero (Prieto et al., 2014).

En España, el transporte de residuos por carretera supone un $6,83 \%$ del peso total de las mercancías transportadas, correspondiendo a los residuos urbanos y asimilables un 2,06\% (Eurostat, 2016).

Los aspectos logísticos asociados a la recogida y el transporte de los residuos son esenciales y condicionan enormemente el coste y los impactos de la gestión de los residuos. En la medida en que se optimice dicha logística, la contención de los costes y los ahorros pueden ser sustanciales (MAGRAMA, 2013).

El transporte de RU supone más de un 50\% de los costes de gestión de los RU (Abengoa, 2007; Transrsu, 2016; Casas et al., 2005), actuar sobre este proceso es fundamental para hacer más eficiente la gestión de los RU y conseguir una reducción del coste que soportan los ciudadanos a la vez que se consiguen mejoras ambientales.

Entre las mejoras tecnológicas que se están implantando en los servicios de recogida podemos citar los sistemas GPS, el pesaje embarcado o en chasis, los sistemas de lectura de tags, y los medidores volumétricos de contenedores (Sánchez, 2015). La aplicación de estas tecnologías, la utilización de camiones bicompartimentados (para la recogida simultánea de varias fracciones), junto a la agrupación de municipios para la prestación del servicio, consigue la optimización de rutas, facilitan el control al prestador del servicio, abaratan los costes que deben soportar los ciudadanos y mejoran las condiciones ambientales del entorno (Lombrano, 2009). 


\section{MATERIALES Y MÉTODOS}

\subsection{Introducción}

La metodología consiste en comparar económica y ambientalmente las mejoras que se obtienen al aplicar las medidas de mancomunación y nuevas tecnologías en dos territorios del Sur-Este español.

La mejora económica consiste en calcular, para cada territorio, el coste por tonelada de recogida y transporte de RU cuando el servicio se realiza de forma individualizada ( $\mathrm{Ci}$, como media ponderada del coste de cada uno de los municipios) y cuando se realiza de forma mancomunada $(\mathrm{Cm})$, siendo la diferencia entre estos dos costes el beneficio económico obtenido por la mancomunación del servicio.

La mejora ambiental consiste en calcular, para cada territorio, las emisiones de $\mathrm{CO}_{2 \text { eq }}$ por tonelada de RU generada, derivadas de la recogida y transporte de RU cuando el servicio se realiza de forma individualizada (Ei) y mancomunada (Em). Igualmente la diferencia nos proporcionará el beneficio ambiental que supone la mancomunación de rutas de recogida y transporte.

Se tendrá en cuenta que las emisiones derivadas de la recogida $\left(E_{R}\right)$ dependen del tiempo que los camiones recolectores de carga lateral o trasera emplean en levantar la totalidad de contenedores de un territorio $\left(t_{R}\right.$ CL y $\left.t_{R} C T\right)$. Por otro lado, las emisiones derivadas del transporte $\left(\mathrm{E}_{\mathrm{T}}\right)$ dependerán del tiempo que los camiones recolectores emplean en circular por carreteras urbanas $\left(\mathrm{t}_{\mathrm{TU}}\right)$, por carreteras interurbanas $\left(\mathrm{t}_{\mathrm{TI}}\right)$ y por autovías $\left(\mathrm{t}_{\mathrm{TA}}\right)$ para la totalidad de rutas de un territorio. La contenerización, y por tanto el sistema de carga de los camiones (Lateral o Trasera), se considera en el cálculo del tiempo de recogida $\left(t_{R}\right.$ CL y $t_{R}$ CT).

Para el análisis ambiental los resultados se calculan en términos de reducción de emisiones de GEI en $\mathrm{KgCO}_{2 \text { eq }} / \mathrm{Tn}$ (Moliner et al., 2011). Cabe destacar que cuando hablamos de gases de efecto invernadero (GEI) nos referimos a $\mathrm{CO}_{2}$ equivalente $\left(\mathrm{CO}_{2 \mathrm{eq}}\right)$, que incluye los seis gases de efecto invernadero recogidos en el Protocolo de Kioto (OCCC, 2013; Ihobe, 2013): dióxido de carbono $\left(\mathrm{CO}_{2}\right)$, metano $\left(\mathrm{CH}_{4}\right)$, óxido de nitrógeno $\left(\mathrm{N}_{2} \mathrm{O}\right)$, hidrofluorocarburos (HFC), perfluorocarburos (PFC) y hexafluoruro de azufre $\left(\mathrm{SF}_{6}\right)$.

En la gestión de residuos, las emisiones de GEI son el resultado de la suma de las emisiones derivadas de la recogida, transporte, transferencia, triaje y tratamiento final de los residuos y emisiones (Generalitat de Catalunya y OCCC, 2016). En nuestro caso, consideraremos sólo las dos primeras ya que son las susceptibles al cambio con la mancomunación del servicio de recogida y transporte de RU de competencia municipal.

La metodología propuesta se va aplicar en el territorio 1 (del que se conocen las características en la situación inicial -en la que el servicio de recogida y transporte de RU se realizaba de manera individualizada- y en la situación actual -en la que el servicio se ha mancomunado-) determinándose el ahorro económico y ambiental que ha supuesto la mancomunación del servicio.

En base a los resultados reales que se desprenden de esta investigación para el territorio 1, se estimará el beneficio económico y ambiental que supondrá la futura mancomunación del servicio de recogida y transporte de RU en el territorio 2 (del que solo se conocen las 
características en la situación actual -en la que el servicio de recogida y transporte de RU se realiza de manera individualizada-).

Se describen a continuación las características principales de los dos territorios considerados (territorio 1 y 2) y el desarrollo metodológico seguido para la obtención de los beneficios económicos y ambientales que se alcanzan con la mancomunación e incorporación de nuevas tecnologías en los servicios de recogida y transporte de RU.

Puesto que el clima que se desarrolla en los dos territorios estudiados es mediterráneo e implica la recogida diaria de contenedores (para evitar procesos de fermentación de la materia orgánica), en esta investigación no se van a tener en cuenta los beneficios económicos y ambientales ocasionados por la implantación de sensores de llenado y manipulación en los contenedores.

\subsection{Análisis previo}

Se analizan dos territorios. En el primero (territorio 1) se realiza la recogida y transporte de RU de forma mancomunada desde hace 3 años y contando con una serie de medidas tecnológicas implantadas que permiten el control y la gestión efectiva de las flotas de recogida. Entre estas medidas caben destacar: la incorporación de GPS y sistemas de pesaje en chasis de los camiones recolectores, chips RFID y TAGs en contenedores, y el desarrollo de un visor online que permite obtener datos en tiempo real de las rutas de recogida que se están desarrollando.

El territorio 2, realiza actualmente la recogida y transporte de RU de forma individualizada pero está prevista la licitación para la recogida conjunta de los RU generados, por lo que se espera que a corto plazo el servicio se agrupe.

\begin{tabular}{|c|c|c|c|}
\hline Territorio & Nombre & Situación & $\begin{array}{c}\text { Sistema actual de } \\
\text { recogida y transporte de } \\
\text { RU }\end{array}$ \\
\hline 1 & $\begin{array}{c}\text { Consorcio Para el } \\
\text { Desarrollo de la } \\
\text { Vega Sierra Elvira } \\
(\text { CVSE) }\end{array}$ & Granada & $\begin{array}{c}\text { Mancomunado + Nuevas } \\
\text { tecnologías }\end{array}$ \\
\hline 2 & $\begin{array}{c}\text { Consorcio Vega Baja } \\
(\mathrm{CVB})\end{array}$ & Alicante & Individualizado \\
\hline
\end{tabular}

Tabla 1 - Localización del territorio 1 y 2 y sistema actual de recogida y transporte.

Los escenarios de este estudio van a ser los siguientes:

- Escenario "i": en el que la recogida y transporte de RU se realiza de forma individualizada.

- Escenario "m": en el que la recogida y transporte de RU se realiza de forma mancomunada.

Consecuentemente, el CVSE (Granada) se encuentra actualmente desarrollando el escenario "m", mientras que se prevé la futura incorporación a este mismo escenario en el CVB 
(Alicante). De momento, en el CVB (Alicante) el escenario implantado es el escenario "i".

\begin{tabular}{|c|c|c|}
\hline Escenario & $\begin{array}{c}\text { Sistema de recogida y } \\
\text { transporte de RU }\end{array}$ & $\begin{array}{c}\text { Territorio que lo desarrolla } \\
\text { actualmente }\end{array}$ \\
\hline $\mathrm{i}$ & Individualizado & $\mathrm{CVB}$ \\
\hline $\mathrm{m}$ & $\begin{array}{c}\text { Mancomunado + Nuevas } \\
\text { tecnologías }\end{array}$ & CVSE \\
\hline
\end{tabular}

Tabla 2 - Escenarios considerados en el estudio.

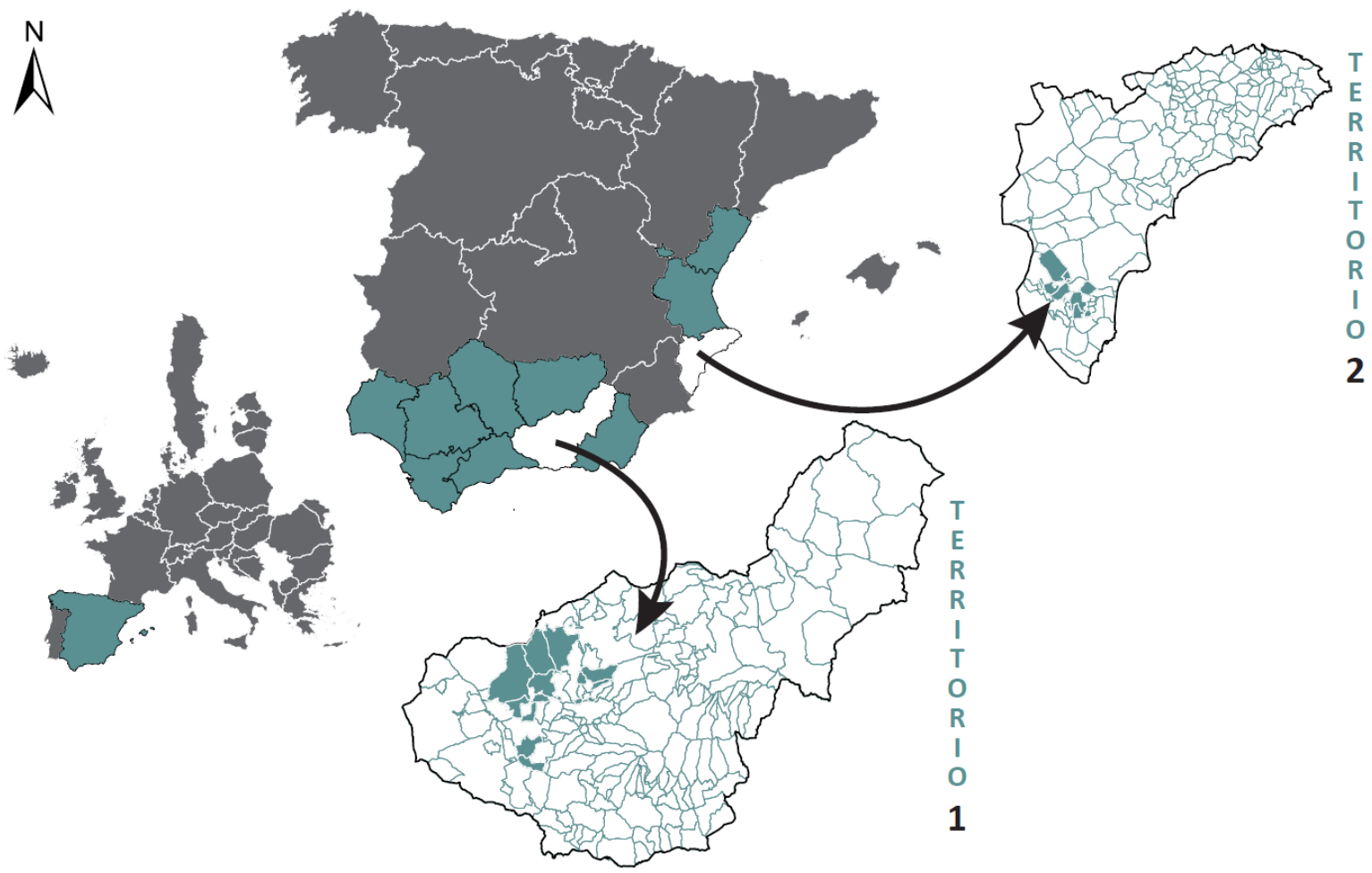

Figura 1 - Situación y emplazamiento del territorio 1 y territorio 2.

\section{3 Ámbito de estudio}

\subsubsection{Territorio 1. Consorcio para el Desarrollo de la Vega Sierra Elvira, CVSE (Granada)}

El CVSE se sitúa al Sur de la Península Ibérica, en la provincia de Granada. Está constituido por 24 municipios de los cuales, se agruparon para la prestación del servicio de recogida un conjunto de 14, con una población total de 66.000 habitantes (INE, 2015) y una generación anual de RU de 31.634,49 Tn/año (Resur Granada, 2012a).

El sistema inicial de recogida y transporte de RU era individualizado, con la existencia de 15 rutas distintas de recogida cuyo destino final era, en su mayoría, la Planta de Tratamiento Mecánico Biológico y vertedero (PTMB+V) de la "Loma de Manzanares" situada en el municipio de Alhendín (Granada). Tan solo 3 de las 15 rutas iniciales finalizaban en una de las 9 Plantas de Transferencia que en la actualidad existen en la provincia de Granada (PT de Montefrío) (Resur Granada, 2012b).

Con la mancomunación del servicio de recogida y transporte, el número de rutas se ha reducido a 10, siendo igualmente el destino final de los residuos, bien la PTMB+V de 
Alhendín o la PT de Montefrío. La reducción del número de rutas ha sido posible ya que el sistema de pesaje en chasis permite determinar la cantidad de RU recogidos en cada municipio, al contrario que en el escenario "i", en el que cada municipio llevaba directamente sus RU bien a la PTMB+V o bien a la PT.

Por lo que respecta al sistema de contenerización, en el escenario individualizado predominaban los contenedores de Carga Trasera, mientras que con la mancomunación del servicio la gran mayoría de los contenedores se han cambiado a Carga Lateral, lo que influye en los tiempos de recogida y transporte urbano.

\begin{tabular}{|c|c|c|c|c|c|}
\hline Territorio & $\begin{array}{c}\text { Número de } \\
\text { municipios }\end{array}$ & $\begin{array}{c}\text { Número } \\
\text { de } \\
\text { habitantes }\end{array}$ & $\begin{array}{c}\text { Generación } \\
\text { anual de } \\
\text { RU } \\
\text { (Tn/año) }\end{array}$ & $\begin{array}{c}\text { Número rutas } \\
\text { escenario } \\
\text { individualizado }\end{array}$ & $\begin{array}{c}\text { Número rutas } \\
\text { escenario } \\
\text { mancomunado }\end{array}$ \\
\hline 1 & 14 & 66.000 & $31.634,49$ & 15 & 10 \\
\hline
\end{tabular}

Tabla 3 - Principales características del territorio 1.

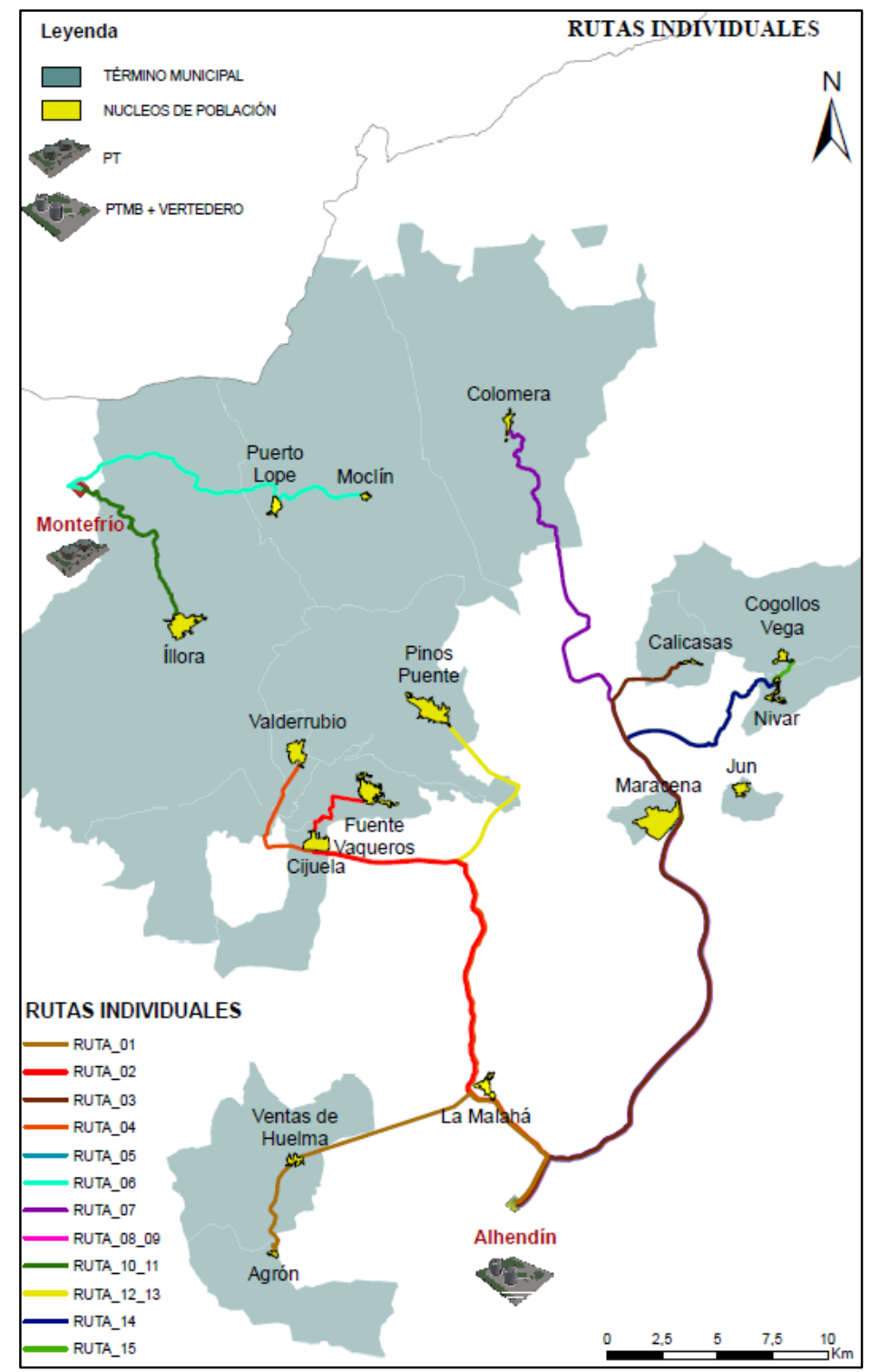

Figura 2 - Rutas individualizadas en el territorio 1. 


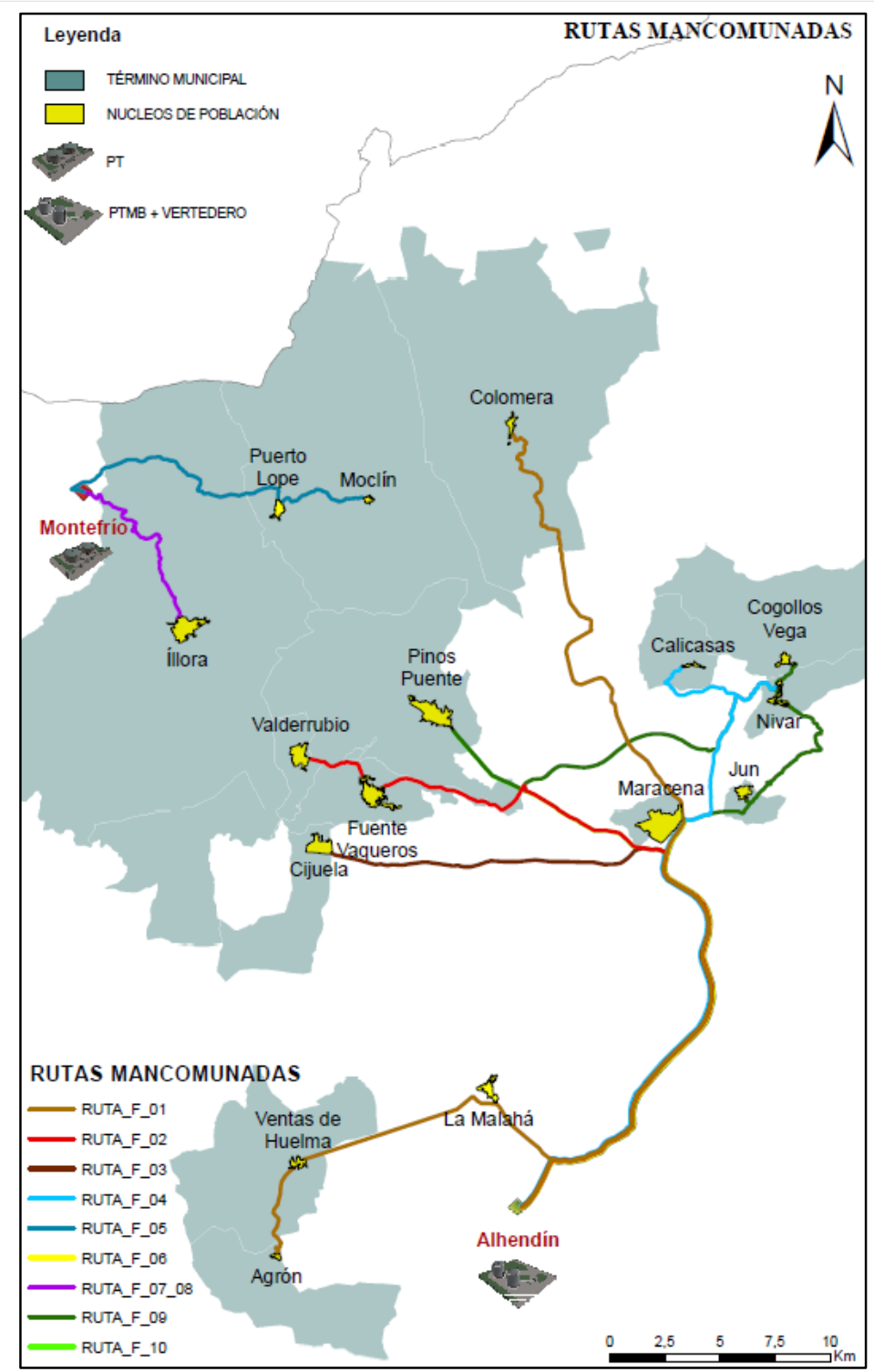

Figura 3 - Rutas mancomunadas en el territorio 1.

\subsubsection{Costes y generación de residuos}

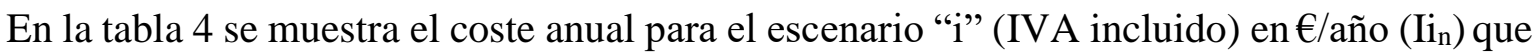
soportaba cada municipio del CVSE (Granada) previamente a la mancomunación del servicio, así como las toneladas anuales de RU (fracción orgánica y resto) generadas en cada uno de ellos en Tn/año $\left(G_{n}\right)$.

El coste anual ( $€ /$ Tn) de la recogida y transporte de RU se ha obtenido de los contratos que cada Ayuntamiento pagaba al concesionario del servicio, mientras que la generación anual de residuos y por tanto la cantidad a transportar (Tn/año), se deduce de las facturas que la $\mathrm{PTMB}+\mathrm{V}$ emite para cada municipio, en función de las toneladas que realmente entran a la $\mathrm{PTMB}+\mathrm{V}$, coincidentes con las transportadas.

Se deduce de la tabla 4 que el coste global de la recogida y transporte de RU para los 14 municipios, realizando el servicio de forma individualizada era de $2.437 .324,90 € /$ año, para una generación y transporte de 31.634,49 Tn en su conjunto, siendo por tanto el coste promedio de 77,05€/Tn (IVA incluido). 


\begin{tabular}{|c|c|c|c|}
\hline $\mathrm{n}$ & Municipio & $\mathrm{Ii}_{\mathrm{n}}(€ / \mathrm{año})$ & $\mathrm{G}_{\mathrm{n}}(\mathrm{Tn} / \mathrm{a} \tilde{n} \mathrm{o})$ \\
\hline 1 & Agrón & $10.823,04$ & 155,52 \\
\hline 2 & Calicasas & $42.528,82$ & 244,49 \\
\hline 3 & Cijuela & $106.168,02$ & $1.513,36$ \\
\hline 4 & Cogollos Vega & $89.922,14$ & $1.413,77$ \\
\hline 5 & Colomera & $46.006,08$ & 669,28 \\
\hline 6 & Fuente Vaqueros & $145.897,98$ & $2.389,27$ \\
\hline 7 & Íllora & $374.653,04$ & $4.969,28$ \\
\hline 8 & Jun & $182.870,37$ & $1.713,60$ \\
\hline 9 & Maracena & $694.930,89$ & $8.497,20$ \\
\hline 10 & Moclín & $183.600,00$ & $1.625,29$ \\
\hline 11 & Nívar & $49.187,79$ & 375,94 \\
\hline 12 & Pinos Puente & $388.949,79$ & $6.116,58$ \\
\hline 13 & Valderrubio & $96.555,81$ & $1.547,13$ \\
\hline 14 & Ventas de Huelma & $25.231,15$ & 403,73 \\
\hline \multicolumn{2}{|r|}{ Total } & $2.437 .324,90$ & $31.634,49$ \\
\hline
\end{tabular}

Tabla 4 - Costes y generación anual de RU en el territorio 1. Escenario individualizado.

\subsubsection{Contenerización}

La tabla 5 recoge la contenerización en el escenario "i” en el CVSE (Granada), determinándose el número de contenedores según el volumen de cada tipo de recipiente. Se observa que la mayor parte de los contenedores eran de Carga Trasera (360 litros, 800 litros y 1.100 litros), $90 \%$ del total, mientras que el $10 \%$ de los contenedores eran de Carga Lateral (3.200 litros).

Estos datos fueron recopilados para la preparación del concurso de licitación del servicio de recogida conjunta de residuos, donde se hizo un estudio exhaustivo de la situación inicial en cada uno de los municipios considerados.

\begin{tabular}{|c|c|c|c|c|c|}
\hline $\mathrm{n}$ & Municipio & $\begin{array}{c}\mathrm{N}^{\mathbf{o}} \text { cont. } \\
360 \mathrm{~L}\end{array}$ & $\begin{array}{c}\mathrm{N}^{\mathbf{o}} \text { cont. 800 } \\
\mathrm{L}\end{array}$ & $\begin{array}{c}\mathrm{N}^{\mathbf{o}} \text { cont. } 1100 \\
\mathrm{~L}\end{array}$ & $\begin{array}{c}\mathrm{N}^{\mathbf{o}} \text { cont. 3200 } \\
\mathrm{L}\end{array}$ \\
\hline 1 & Agrón & 7,00 & 8,00 & & \\
\hline 2 & Calicasas & 8,00 & 23,00 & & \\
\hline 3 & Cijuela & 5,00 & 102,00 & & \\
\hline 4 & Cogollos Vega & 5,00 & 53,00 & & \\
\hline 5 & Colomera & 12,00 & 61,00 & & \\
\hline 6 & Fuente Vaqueros & 23,00 & 127,00 & & 57,00 \\
\hline 7 & Íllora & 33,00 & 298,00 & & \\
\hline 8 & Jun & & & & \\
\hline 9 & Maracena & 6,00 & & 388,00 & \\
\hline 10 & Moclín & 140,00 & 107,00 & & 124,00 \\
\hline 11 & Nívar & & 48,00 & & \\
\hline 12 & Pinos Puente & 14,00 & 101,00 & & \\
\hline 13 & Valderrubio & 3,00 & 70,00 & & 181,00 \\
\hline 14 & Ventas de Huelma & 11,00 & 19,00 & & \\
\hline \multicolumn{7}{|c|}{ Total } & 267,00 & $1.017,00$ & 388,00 & \\
\hline
\end{tabular}

Tabla 5 - Contenerización en el territorio 1. Escenario individualizado. 
Con la mancomunación del servicio en el CVSE (Granada) la mayoría de los contenedores de Carga Trasera (360 litros, 800 litros y 1.100 litros) se han sustituido por contenedores de Carga Lateral (3.200 litros) en correspondencia con los datos representados en la tabla 6.

Entre las ventajas del cambio a Carga Lateral se pueden citar las siguientes (Calvo et al., 1997): Menores costes, mayor seguridad en los operarios, operación más limpia, menor ocupación de la vía pública, menor nivel sonoro, etc.

Los datos de la contenerización en el escenario " $\mathrm{m}$ " son conocidos ya que se ha implantado la contenerización propuesta en la oferta del gestor adjudicatario de los servicios mancomunados de recogida y transporte de RU.

\begin{tabular}{|c|c|c|c|c|c|}
\hline $\mathrm{n}$ & Municipio & $360 \mathrm{~L}$ & $800 \mathrm{~L}$ & $1100 \mathrm{~L}$ & $3200 \mathrm{~L}$ \\
\hline 1 & Agrón & 7,00 & 8,00 & & \\
\hline 2 & Calicasas & & & & 12,00 \\
\hline 3 & Cijuela & & & & 39,00 \\
\hline 4 & Cogollos Vega & & & & 18,00 \\
\hline 5 & Colomera & & & & 22,00 \\
\hline 6 & Fuente Vaqueros & & & & 49,00 \\
\hline 7 & Íllora & 40,00 & 20,00 & & 111,00 \\
\hline 8 & Jun & & & & 57,00 \\
\hline 9 & Maracena & 6,00 & & 266,00 & 85,00 \\
\hline 10 & Moclín & 9,00 & 9,00 & & 88,00 \\
\hline 11 & Nívar & & & & 18,00 \\
\hline 12 & Pinos Puente & 14,00 & 101,00 & & 124,00 \\
\hline 13 & Valderrubio & & & & 32,00 \\
\hline 14 & Ventas de Huelma & 11,00 & 19,00 & & \\
\hline \multicolumn{7}{|c|}{ Total } & 87,00 & 157,00 & 266,00 & 655,00 \\
\hline
\end{tabular}

Tabla 6 - Contenerización en el territorio 1. Escenario mancomunado.

\subsubsection{Tiempos de rutas}

En la tabla 7 se recogen los tiempos de las 15 rutas de recogida y transporte de RU en el CVSE (Granada) del escenario "i” y los tiempos diarios (horas/día) empleados en cada ruta distinguiendo:

- Tiempo urbano $\left(t_{U}\right)$ : incluye el tiempo de recogida $\left(t_{R}\right)$, empleado en la levantada de contenedores, más el tiempo de transporte urbano entre contenedor y contendor $\left(\mathrm{t}_{\mathrm{TU}}\right)$.

- Tiempo de transporte interurbano $\left(\mathrm{t}_{\mathrm{TI}}\right)$ : que corresponde con el tiempo de transporte por carretera convencional entre municipio y municipio y/o entre base de salida y municipio inicial de recogida.

- Tiempo de transporte por autovía ( $\left.\mathrm{t}_{\mathrm{TA}}\right)$ : tiempo de transporte por autovía empleado en la ida desde el último municipio recogido hasta la PTMB+V o hasta la PT (es decir, hasta el punto de descarga) y la vuelta desde estos puntos de descarga hasta el municipio inicial o base de salida. 


\begin{tabular}{|c|c|c|c|c|}
\hline Id & Ruta & $\begin{array}{c}\text { Tiempo urbano } \\
t_{U n}=t_{R n+} t_{T U n}\end{array}$ & $\begin{array}{c}\text { Tiempo } \\
\text { transporte } \\
\text { (interurbano) } \\
\mathrm{t}_{\text {TIn }} \\
\end{array}$ & $\begin{array}{c}\text { Tiempo } \\
\text { transporte } \\
\text { (autovía) } \\
\mathrm{t}_{\mathrm{TAn}}\end{array}$ \\
\hline \multirow{2}{*}{ r1 } & \multirow{2}{*}{ Agrón-Ventas de Huelma } & 0:22:00 & \multirow{2}{*}{$0: 21: 26$} & \multirow{2}{*}{$0: 18: 40$} \\
\hline & & $0: 48: 00$ & & \\
\hline \multirow{2}{*}{$\mathrm{r} 2$} & \multirow{2}{*}{ Fuente Vaqueros-Cijuela } & $1: 55: 00$ & \multirow{2}{*}{ 0:10:00 } & \multirow{2}{*}{$0: 48: 44$} \\
\hline & & 1:30:00 & & \\
\hline r3 & Calicasas & $0: 36: 00$ & & $0: 58: 06$ \\
\hline $\mathrm{r} 4$ & Valderrubio & 1:18:00 & & 1:00:51 \\
\hline $\mathrm{r} 5$ & Jun CL & 2:00:00 & & $0: 45: 05$ \\
\hline r6 & Moclín & $4: 15: 00$ & $1: 16: 28$ & $0: 19: 12$ \\
\hline $\mathrm{r} 7$ & Colomera & $4: 15: 00$ & & $1: 21: 36$ \\
\hline $\mathrm{r} 8$ & Maracena 1 & $3: 30: 00$ & & $0: 41: 49$ \\
\hline r9 & Maracena 2 & $3: 30: 00$ & & $0: 41: 49$ \\
\hline $\mathrm{r} 10$ & Íllora 1 & $4: 26: 00$ & $0: 24: 03$ & $0: 32: 34$ \\
\hline r11 & Íllora 2 & $4: 27: 00$ & $0: 25: 00$ & $0: 27: 41$ \\
\hline $\mathrm{r} 12$ & Pinos Puente CL & $3: 43: 00$ & $1: 15: 19$ & $0: 48: 20$ \\
\hline $\mathrm{r} 13$ & Pinos Puente CT & 4:05:00 & $0: 25: 00$ & $0: 48: 20$ \\
\hline $\mathrm{r} 14$ & Nívar & 1:00:00 & & $1: 04: 27$ \\
\hline $\mathrm{r} 15$ & Cogollos Vega & 2:00:00 & & $1: 07: 53$ \\
\hline & Total (horas/día) & 43:40:00 & $4: 17: 16$ & $11: 45: 07$ \\
\hline
\end{tabular}

Tabla 7 - Rutas y tiempos en el territorio 1. Escenario individualizado.

Se deduce de la tabla 7 que el mayor tiempo de ruta en la situación inicial se corresponde con el tiempo urbano, seguido del tiempo de transporte por autovía y por último, el tiempo interurbano. Se aprecia que en determinadas rutas el tiempo interurbano es igual a cero, lo cual se traduce en que esa ruta realizaba la recogida y transporte individualizada al $100 \%$, sin embargo, las rutas con tiempo interurbano distinto de cero se corresponden con aquellas que integraban a otros núcleos de población o bien, aquellos municipios que recogen una o varias pedanías de tamaño considerable.

La mancomunación del servicio en el CVSE (Granada) ha supuesto que las 15 rutas iniciales de recogida y transporte de la fracción orgánica y resto se hayan reducido a 10 rutas. Con la implementación de nuevas tecnologías al servicio y el apoyo de un Visor online que permite visualizar datos en tiempo real, se pueden conocer entre otros, los tiempos de recogida y transporte diarios que describe cada ruta (horas/día) mostrados en la tabla 8. 


\begin{tabular}{|c|c|c|c|c|}
\hline Id & Ruta & $\begin{array}{l}\text { Tiempo urbano } \\
t_{U n}=t_{R n+} t_{T U n}\end{array}$ & $\begin{array}{c}\text { Tiempo } \\
\text { transporte } \\
\text { (interurbano) } \\
\text { t }_{\text {TIn }}\end{array}$ & $\begin{array}{c}\text { Tiempo } \\
\text { transporte } \\
\text { (autovía) } \\
\mathrm{t}_{\mathrm{TAn}}\end{array}$ \\
\hline \multirow{3}{*}{$\mathrm{r} 1$} & \multirow{3}{*}{$\begin{array}{c}\text { Colomera-Ventas de Huelma- } \\
\text { Agrón }\end{array}$} & $1: 47: 11$ & \multirow{3}{*}{$1: 22: 17$} & \multirow{3}{*}{$2: 40: 20$} \\
\hline & & $0: 53: 16$ & & \\
\hline & & $0: 17: 59$ & & \\
\hline \multirow{2}{*}{$\mathrm{r} 2$} & \multirow{2}{*}{ Valderrubio-Fuente Vaqueros } & $2: 18: 34$ & \multirow{2}{*}{$0: 30: 15$} & \multirow{2}{*}{ 1:07:06 } \\
\hline & & $1: 15: 29$ & & \\
\hline r3 & Cijuela & $2: 19: 36$ & $0: 10: 27$ & $0: 40: 56$ \\
\hline \multirow{3}{*}{$\mathrm{r} 4$} & \multirow{3}{*}{ Maracena-Nívar-Calicasas } & $0: 42: 31$ & \multirow{3}{*}{$0: 40: 26$} & \multirow{3}{*}{$1: 31: 49$} \\
\hline & & $0: 51: 28$ & & \\
\hline & & $3: 25: 46$ & & \\
\hline r5 & Moclín & $3: 18: 14$ & $1: 19: 55$ & $0: 31: 45$ \\
\hline r6 & Maracena CT & $6: 23: 32$ & $0: 00: 00$ & $1: 26: 33$ \\
\hline $\mathrm{r} 7$ & Íllora CL & $4: 32: 00$ & $0: 37: 00$ & $0: 40: 00$ \\
\hline r8 & Íllora CT & 2:08:00 & $0: 24: 03$ & 0:40:00 \\
\hline \multirow{3}{*}{ r9 } & \multirow{3}{*}{$\begin{array}{c}\text { Jun-Cogollos Vega-Pinos Puente } \\
\text { CL }\end{array}$} & $1: 30: 00$ & \multirow{3}{*}{ 0:46:04 } & \multirow{3}{*}{ 0:46:06 } \\
\hline & & $1: 24: 00$ & & \\
\hline & & $4: 12: 00$ & & \\
\hline $\mathrm{r} 10$ & Pinos Puente CT & $1: 54: 00$ & $0: 25: 00$ & $0: 46: 06$ \\
\hline & Total (horas/día) & $39: 13: 36$ & $6: 15: 27$ & $10: 50: 41$ \\
\hline
\end{tabular}

Tabla 8 - Rutas y tiempos en el territorio 1. Escenario mancomunado.

Coincidiendo con el escenario "i", en el escenario actual " $m$ ", el mayor tiempo de ruta se corresponde con el tiempo urbano, seguido del tiempo de transporte por autovía y finalmente el tiempo de transporte interurbano. Se observa que en esta nueva situación, el tiempo urbano ha disminuido respecto al tiempo urbano inicial indicado en la tabla 7. Esto se debe a que hay un menor número de contenedores que recoger, pues el cambio de Carga Trasera a Carga Lateral implica un menor número de recipientes instalados, por un aumento de volumen del contenedor.

Respecto al tiempo de transporte por autovía, en la situación mancomunada ha disminuido respecto a la situación inicial, ya que el número de rutas ha disminuido.

Finalmente, el tiempo de transporte interurbano ha aumentado en la situación actual respecto a la situación inicial pues la mancomunación, implica un mayor número de pueblos y pedanías contenidos en una misma ruta.

\subsubsection{Territorio 2. Consorcio de la Vega Baja, CVB (Alicante)}

El Consorcio de la Vega Baja (CVB) se sitúa al Sur-Este de la Península Ibérica, en la provincia de Alicante. Está constituido por un conjunto de 27 municipios de los que se han simulado para la recogida conjunta un total de 8 con una población total de 74.144 habitantes (INE, 2015) y una generación anual de RU de 34.981,42 Tn/año según datos de 2015.

El sistema actual de recogida y transporte de RU es individualizado, con la existencia de 11 rutas distintas de recogida cuyo destino final es, bien la Planta de Tratamiento Mecánico Biológico y vertedero $(\mathrm{PTMB}+\mathrm{V})$ de Jijona o bien la Planta de Tratamiento Mecánico Biológico y vertedero (PTMB+V) de Villena, ambas en la provincia de Alicante.

Se espera que en un futuro próximo algunos municipios del territorio 2 se agrupen para 
realizar el servicio de recogida y transporte de RU.

\begin{tabular}{|c|c|c|c|c|c|}
\hline Territorio & $\begin{array}{c}\text { Número de } \\
\text { municipios }\end{array}$ & $\begin{array}{c}\text { Número de } \\
\text { habitantes }\end{array}$ & $\begin{array}{c}\text { Generación } \\
\text { anual de } \\
\text { RU } \\
\text { (Tn/año) }\end{array}$ & $\begin{array}{c}\text { Número } \\
\text { rutas } \\
\text { actuales }\end{array}$ & $\begin{array}{c}\text { Número rutas } \\
\text { futuras, tras } \\
\text { mancomunación }\end{array}$ \\
\hline 2 & 8 & 74.144 & $34.981,42$ & 11 & sd \\
\hline
\end{tabular}

Tabla 9 - Principales características del territorio 2.

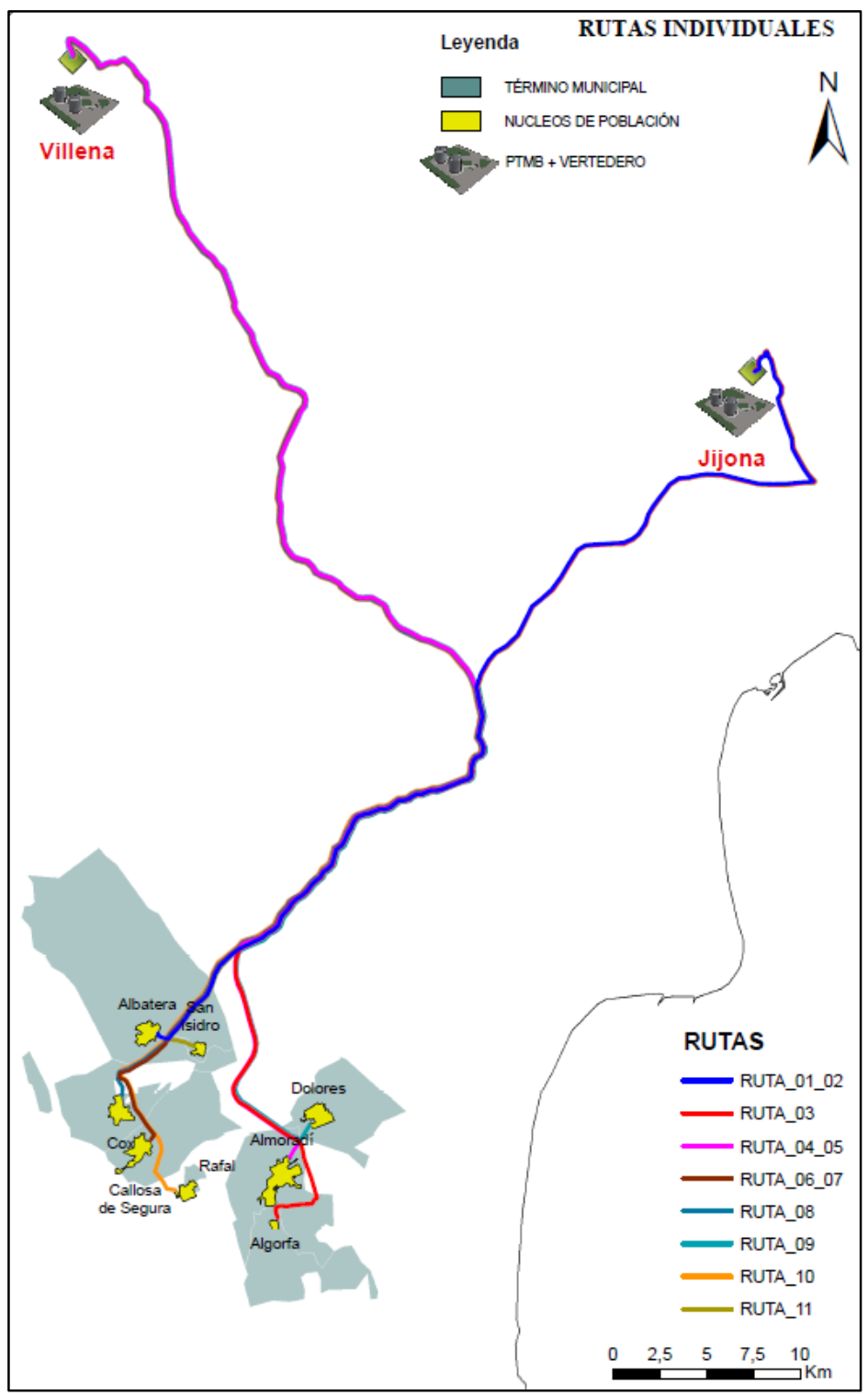

Figura 4 - Rutas individualizadas en el territorio 2.

\subsubsection{Costes y generación de residuos}

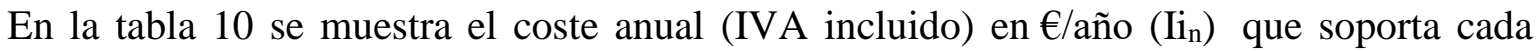
municipio del CVB (Alicante) así como las toneladas anuales de RU generadas (Tn/año) en cada uno de ellos (fracción orgánica y resto).

La obtención del coste anual (€/Tn) de la recogida y transporte de RU así como la generación 
anual de residuos (Tn/año) se obtiene con las mismas consideraciones indicadas en el apartado 2.2.1.

Se deduce de la tabla 10 que el coste global de la recogida y transporte de RU para los 8 municipios, realizando el servicio de forma individualizada era de $1.971 .475,05 €$ /año para una generación de $34.981,42$ Tn en su conjunto, siendo por tanto el coste promedio de 56,36 $€ /$ Tn (IVA incluido).

\begin{tabular}{|c|c|c|c|}
\hline $\mathrm{n}$ & Municipio & $\mathrm{Ii}_{\mathrm{n}}(€ /$ año $)$ & $\mathrm{G}_{\mathrm{n}}(\mathrm{Tn} / \mathrm{año})$ \\
\hline 1 & Albatera & $295.293,14$ & $6.420,00$ \\
\hline 2 & Algorfa & $156.280,09$ & $1.951,00$ \\
\hline 3 & Almoradí & $413.895,35$ & $8.030,00$ \\
\hline 4 & Callosa & $473.041,38$ & $7.935,00$ \\
\hline 5 & Cox & $196.200,00$ & $3.937,54$ \\
\hline 6 & Dolores & $191.928,00$ & $3.594,71$ \\
\hline 7 & Rafal & $127.837,08$ & $1.912,17$ \\
\hline 8 & San Isidro & $117.000,00$ & $1.201,00$ \\
\hline & Total & $1.971 .475,05$ & $34.981,42$ \\
\hline
\end{tabular}

Tabla 10 - Costes y generación anual de RU en el territorio 2. Escenario individualizado.

\subsubsection{Contenerización}

La tabla 11 recoge la contenerización actual para el escenario "i" en el CVB (Alicante), determinándose el número de contenedores según el volumen de cada tipo de recipiente. Se observa que la totalidad de los recipientes son de Carga Trasera (240, 800 y 1.100 litros).

\begin{tabular}{|c|c|c|c|}
\hline $\mathrm{n}$ & Municipio & $\mathrm{N}^{\mathbf{o}}$ cont. 240 L & $\mathrm{N}^{\mathbf{0}}$ cont. 800 - 1.100 L \\
\hline 1 & Albatera & 3 & 396 \\
\hline 2 & Algorfa & & 169 \\
\hline 3 & Almoradí & & 487 \\
\hline 4 & Callosa & 100 & 560 \\
\hline 5 & Cox & & 362 \\
\hline 6 & Dolores & 6 & 386 \\
\hline 7 & Rafal & & 147 \\
\hline 8 & San Isidro & 109 & 86 \\
\hline
\end{tabular}

Tabla 11 - Contenerización en el territorio 2. Escenario individualizado.

En cuanto a la contenerización en el escenario “m”, a priori no se sabe qué tipo será instalado, pero la morfología de los pueblos admiten tanto la Carga Trasera como la Carga Lateral.

\subsubsection{Tiempo de rutas}

En la tabla 12 se recogen las 11 rutas actuales correspondientes con el escenario "i" de recogida y transporte de RU en el CVB (Alicante) así como los tiempos diarios empleados (horas/día) en cada ruta distinguiendo igualmente los tres tiempos comentados en el apartado 2.2.3.

Estos datos se conocen del estudio realizado de forma previa al concurso de licitación para 
la gestión de forma mancomunada del servicio de recogida y transporte de RU que está actualmente en curso.

\begin{tabular}{|c|c|c|c|c|}
\hline Id & Ruta & $\begin{array}{c}\text { Tiempo urbano } \\
\mathrm{t}_{\text {Un }} \mathrm{t}_{\mathrm{Rn}+} \mathrm{t}_{\mathrm{TUn}}\end{array}$ & $\begin{array}{c}\text { Tiempo } \\
\text { transporte } \\
\text { (interurbano) } \\
\mathrm{t}_{\mathrm{T} \text { In }}\end{array}$ & $\begin{array}{c}\text { Tiempo } \\
\text { transporte } \\
\text { (autovía) } \\
\mathrm{t}_{\mathrm{TAn}}\end{array}$ \\
\hline r1 & Albatera & $4: 00: 00$ & & $1: 29: 09$ \\
\hline r2 & Albatera 2 & $4: 00: 00$ & & $1: 29: 09$ \\
\hline r3 & Algorfa & $2: 49: 39$ & $0: 10: 21$ & $1: 53: 09$ \\
\hline r4 & Almoradí & $5: 24: 00$ & & $2: 13: 43$ \\
\hline r5 & Almoradí 2 & $5: 24: 00$ & & $2: 13: 43$ \\
\hline r6 & Callosa de Segura & $5: 56: 00$ & & $2: 06: 51$ \\
\hline r7 & Callosa de Segura 2 & $5: 56: 00$ & & $2: 06: 51$ \\
\hline r8 & Cox & $6: 22: 00$ & & $2: 10: 27$ \\
\hline r9 & Dolores & $6: 33: 00$ & & $2: 06: 21$ \\
\hline r10 & Rafal & $3: 00: 00$ & & $1: 18: 31$ \\
\hline r11 & San Isidro & $2: 30: 00$ & $0: 18: 36$ & $21: 20: 03$ \\
\hline \multicolumn{2}{|c|}{ Total (horas/día) } & $51: 54: 39$ & $0: 28: 57$ & \\
\hline
\end{tabular}

Tabla 12 - Rutas y tiempos en el territorio 2. Escenario individualizado.

Se deduce de la tabla 12 que el mayor tiempo de ruta se corresponde con el tiempo urbano, seguido del tiempo de transporte por autovía y por último, el tiempo interurbano. Se aprecia que en determinadas rutas el tiempo interurbano es igual a cero, lo cual se traduce en que esa ruta realiza la recogida y transporte individualizada al 100\%, sin embargo, las rutas con tiempo interurbano distinto de cero se corresponden con aquellas que integran a otros núcleos de población o bien, aquellos municipios que recogen una o varias pedanías de tamaño considerable.

Se espera que el escenario "m", el número de rutas actuales se vea reducido al menos a 9 rutas, por lo que es previsible que el tiempo de transporte interurbano aumente respecto al escenario "i" y el tiempo de transporte por autovía disminuya respecto a la situación actual por los mismos motivos que se han comentado en el apartado 2.2.3.

\subsection{Cálculos análisis económico}

Se calcula el beneficio obtenido por la mancomunación del servicio de recogida y transporte de $R U$, en $€ / T n$ de $R U$ generada anualmente, mediante la diferencia entre el coste del servicio en el escenario individualizado $(\mathrm{Ci})$ y el coste del servicio en el escenario mancomunado $(\mathrm{Cm})$.

Siendo,

$$
\text { Beneficio Económico }\left(\frac{€}{T n}\right)=C i\left(\frac{€}{T n}\right)-C m\left(\frac{€}{T n}\right)
$$

- Ci: costes del servicio de recogida y transporte de RU en el escenario individualizado $(€ / \mathrm{Tn})$.

- $\mathrm{Cm}$ : costes del servicio de recogida y transporte de RU en el escenario mancomunado $(€ / \mathrm{Tn})$. 


\subsubsection{Coste del servicio en el escenario individualizado, $(\mathrm{Ci})$}

Para el coste del servicio en el escenario "i" hay que tener en cuenta:

- Importe anual que cada Ayuntamiento paga al Concesionario del servicio $\left(\mathrm{Ii}_{\mathrm{n}}\right)$ en $€ /$ año.

- Toneladas anuales generadas en el municipio $\left(\mathrm{G}_{\mathrm{n}}\right)$ en $\mathrm{Tn} / \mathrm{año}$.

El importe anual relativo a cada municipio se obtiene a partir de las 12 facturas correspondientes con cada mes del año y que cada Ayuntamiento paga al concesionario encomendado de los servicios de recogida y transporte de RU. Las toneladas anuales de RU generadas en cada municipio se obtienen a partir de las facturas emitidas cada mes desde la Planta de Tratamiento Mecánico Biológico a la que se destinan los residuos, puesto que el cobro de estas facturas se realiza en función del pesaje que se registra en la báscula de la Planta, a la entrada de cada camión.

Con estos dos datos se puede calcular el coste del servicio soportado por cada municipio en el escenario individualizado y para el territorio considerado $\left(\mathrm{Ci}_{\mathrm{n}}\right)$ sin más que dividir el importe anual $\left(\mathrm{Ii}_{\mathrm{n}}\right)$ entre las toneladas generadas $\left(\mathrm{G}_{\mathrm{n}}\right)$, esto es:

$$
C i_{n}\left(\frac{€}{T n}\right)=\frac{I i_{n}\left(\frac{€}{a \tilde{o} o}\right)}{G_{n}\left(\frac{T n}{a \tilde{n} o}\right)}
$$

Siendo,

- $\mathrm{Ci}_{\mathrm{n}}$ : Coste del servicio de recogida y transporte de $\mathrm{RU}$, por tonelada generada, para el municipio $n$ en el escenario individualizado $(€ / \mathrm{Tn})$.

- $\quad \mathrm{Ii}_{\mathrm{n}}$ : Importe anual que el Ayuntamiento del municipio $\mathrm{n}$ paga al concesionario encomendado del servicio de recogida y transporte de RU en el escenario individualizado (€/año).

- $\mathrm{G}_{\mathrm{n}}$ : Generación anual de RU en el municipio n (Tn/año).

- $\mathrm{n}$ : número de municipios integrantes del territorio considerado.

Para calcular el coste inicial del conjunto de municipios (Ci) se aplicará:

$$
C i\left(\frac{€}{T n}\right)=\frac{\sum_{n=1}^{n} I i_{n}\left(\frac{€}{a \tilde{n} 0}\right)}{\sum_{n=1}^{n} G_{n}\left(\frac{T n}{a n ̃ o}\right)}
$$

Siendo,

- Ci: Coste del servicio de recogida y transporte de RU, por tonelada generada, para el conjunto de municipios del territorio considerado en el escenario individualizado $(€ / \mathrm{Tn})$.

\subsubsection{Coste del servicio en el escenario mancomunado, $(\mathrm{Cm})$}

Este coste es conocido en el caso del Consorcio para el Desarrollo de la Vega Sierra Elvira, CVSE (Granada), y se extrae de la oferta económica que el adjudicatario del concurso presentó para la encomienda del servicio de recogida y transporte de RU para la implementación del sistema conjunto. 


$$
\text { Cm }(\text { CVSE, Granada })=\text { Conocido de la of erta adjudicatario }=63,90 \frac{€}{T n}
$$

(IVA incluido) (4)

Siendo,

- $\mathrm{C}_{\mathrm{m}}$ (CVSE, Granada): Coste del servicio de recogida y transporte de RU, por tonelada generada, para el conjunto de municipios del territorio 1 (CVSE, Granada) en el escenario mancomunado $(€ / \mathrm{Tn})$.

En el caso del Consorcio de la Vega Baja, CVB (Alicante), este coste no es conocido porque el concurso está actualmente en trámites y a fecha de redacción del presente estudio no ha sido adjudicado. No obstante, en la preparación de los Pliegos de dicho concurso, se incluía un coste del servicio "a mejorar" por el adjudicatario, y que se calculó con los mismos criterios que para el caso del CVSE (Granada). No se conoce por tanto el coste definitivo del servicio para el escenario mancomunado (futuro), pero sí una buena aproximación para este territorio.

$$
\begin{gathered}
C m(C V B, \text { Alicante })= \\
\text { Aproximación Pliegos concurso }=51,19 \frac{€}{T n}
\end{gathered}
$$

Siendo,

- $\mathrm{C}_{\mathrm{m}}$ (CVB, Alicante): Coste del servicio de recogida y transporte de RU, por tonelada generada, para el conjunto de municipios del territorio 2 (CVB, Alicante) en el escenario mancomunado $(€ / \mathrm{Tn})$.

\subsection{Cálculos análisis ambiental}

$\mathrm{El}$ análisis ambiental consiste en calcular para un territorio concreto el beneficio obtenido, en $\mathrm{KgCO}_{2 \text { eq }} / \mathrm{Tn}$ de $\mathrm{RU}$ generada anualmente, a partir de la diferencia de las emisiones derivadas de la recogida y transporte en el escenario individualizado (Ei) y en el escenario mancomunado (Em), esto es:

$$
\text { Beneficio Ambiental }\left(\frac{\mathrm{KgCO}_{2 e q}}{\mathrm{Tn}}\right)=\mathrm{Ei}\left(\frac{\mathrm{KgCO}_{2 e q}}{\mathrm{Tn}}\right)-\mathrm{Em}\left(\frac{\mathrm{KgCO}_{2 e q}}{\mathrm{Tn}}\right)
$$

Siendo,

- Ei: Emisiones derivadas de la recogida y transporte de RU en el escenario individualizado $\left(\mathrm{KgCO}_{2 \mathrm{eq}} / \mathrm{Tn}\right)$.

- Em: Emisiones derivadas de la recogida y transporte de RU en el escenario mancomunado $\left(\mathrm{KgCO}_{2 \mathrm{eq}} / \mathrm{Tn}\right)$.

Las emisiones en el escenario “i”" (Ei) y en el escenario “ $m$ ” (Em) se calculan como sumatoria de las emisiones derivadas de la recogida de $\mathrm{RU}\left(\mathrm{E}_{\mathrm{R}}\right)$ y las emisiones derivadas del transporte $\left(\mathrm{E}_{\mathrm{T}}\right)$ (Generalitat de Catalunya y OCCC, 2016): 


$$
\begin{gathered}
E i\left(\frac{K g O_{2 e q}}{T n}\right)=E_{R i}\left(\frac{K g C O_{2 e q}}{T n}\right)+E_{T i}\left(\frac{K_{C C O} e q}{T n}\right) \\
E m\left(\frac{K g C O_{2 e q}}{T n}\right)=E_{R m}\left(\frac{K g C O_{2 e q}}{T n}\right)+E_{T m}\left(\frac{K g C O_{2 e q}}{T n}\right)
\end{gathered}
$$

Siendo,

- ERi: Emisiones derivadas de la recogida de RU en el escenario individualizado $\left(\mathrm{KgCO}_{2 \mathrm{eq}} / \mathrm{Tn}\right)$.

- $\mathrm{E}_{\mathrm{Ti}}$ : Emisiones derivadas del transporte de RU en el escenario individualizado $\left(\mathrm{KgCO}_{2 \mathrm{eq}} / \mathrm{Tn}\right)$.

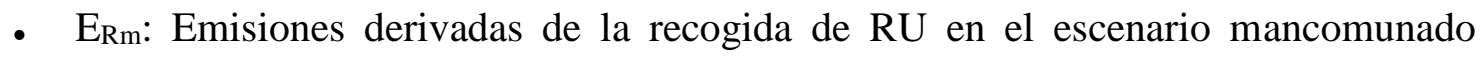
$\left(\mathrm{KgCO}_{2 \mathrm{eq}} / \mathrm{Tn}\right)$.

- $\mathrm{E}_{\mathrm{Tm}}$ : Emisiones derivadas del transporte de RU en el escenario mancomunado $\left(\mathrm{KgCO}_{2 \mathrm{eq}} / \mathrm{Tn}\right)$.

A su vez, las emisiones derivadas del transporte se subdividen en emisiones asociadas al transporte urbano $\left(\mathrm{E}_{\mathrm{TU}}\right)$, emisiones asociadas al transporte interurbano $\left(\mathrm{E}_{\mathrm{TI}}\right)$ y emisiones asociadas al transporte por autovía ( $\left.\mathrm{E}_{\mathrm{TA}}\right)$ :

$$
\begin{gathered}
E_{T i}\left(\frac{K g C O_{2 e q}}{T n}\right)=E_{T U i}\left(\frac{K g C O_{2 e q}}{T n}\right)+E_{T I i}\left(\frac{K g C O_{2 e q}}{T n}\right)+E_{T A i}\left(\frac{K g C O_{2 e q}}{T n}\right) \\
E_{T m}\left(\frac{K g C O_{2 e q}}{T n}\right)=E_{T U m}\left(\frac{K g C O_{2 e q}}{T n}\right)+E_{T I m}\left(\frac{K g C O_{2 e q}}{T n}\right)+E_{T A m}\left(\frac{K g C O_{2 e q}}{T n}\right)
\end{gathered}
$$

Siendo,

- $\mathrm{E}_{\mathrm{TU}}$ : Emisiones derivadas del transporte de RU por carretera urbana en el escenario individualizado $\left(\mathrm{KgCO}_{2 \mathrm{eq}} / \mathrm{Tn}\right)$.

- ETri: Emisiones derivadas del transporte de RU por carretera interurbana en el escenario individualizado $\left(\mathrm{KgCO}_{2 \mathrm{eq}} / \mathrm{Tn}\right)$.

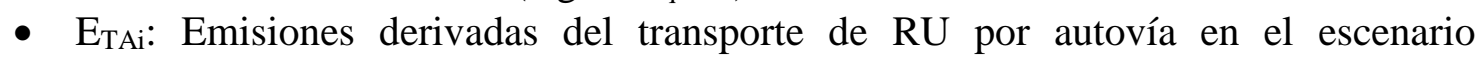
individualizado $\left(\mathrm{KgCO}_{2 \mathrm{eq}} / \mathrm{Tn}\right)$.

- ETUm: Emisiones derivadas del transporte de RU por carretera urbana en el escenario mancomunado $\left(\mathrm{KgCO}_{2 \mathrm{eq}} / \mathrm{Tn}\right)$.

- $\mathrm{E}_{\mathrm{TIm}}$ : Emisiones derivadas del transporte de RU por carretera interurbana en el escenario mancomunado $\left(\mathrm{KgCO}_{2 \mathrm{eq}} / \mathrm{Tn}\right)$.

- $\mathrm{E}_{\mathrm{TAm}}$ : Emisiones derivadas del transporte de RU por autovía en el escenario mancomunado $\left(\mathrm{KgCO}_{2 \mathrm{eq}} / \mathrm{Tn}\right)$.

El desarrollo de cada uno de estos términos para ambos escenarios (" $i$ " o "m") se especifica a continuación. Para su aplicación concreta al escenario "i” o "m”, la única diferencia vendrá dada por los datos a sustituir y que corresponderán, bien con los datos del escenario "i" o bien con los datos del escenario "m".

Hay que tener en cuenta que las emisiones en el escenario " $m$ " para el territorio 2 serán estimadas en base a los resultados que se obtengan del análisis ambiental del territorio 1. 


\subsubsection{Emisiones derivadas de la recogida, $\left(E_{R}\right)$}

Se corresponden con las emisiones del camión recolector de RU cuando se están vaciando los contenedores, es decir, el motor del camión estará encendido pero no se está moviendo el vehículo.

Para determinarlas, en primer lugar se calcula el tiempo diario de ruta destinado a la recogida de RU ( $\left.t_{R}\right)$, considerando que éste se corresponde con el tiempo que el camión emplea en cada una de las levantadas del municipio, sin incluir el tiempo que el camión emplea en desplazarse entre levantada y levantada (este es el tiempo de transporte urbano, $\mathrm{t}_{\mathrm{TU}}$, y se explica en el apartado siguiente).

Conociendo el número de contenedores clasificados por su volumen y el sistema de carga (Trasera o Lateral), el tiempo de recogida $\left(t_{R}\right)$ se obtiene asignando una duración de la levantada que viene determinada en función de estas variables (Fernández, 2012):

- 1,25 min para contenedores de CL (3.200 L)

- 0,55 min para contenedores pequeños de CT $(120 \mathrm{~L}-360 \mathrm{~L})$

- 0,75 min para contenedores grandes de CT $(800 \mathrm{~L}-1.100 \mathrm{~L})$

Es decir, que para un municipio n:

- Tiempo de recogida de contenedores de Carga Lateral en el municipio n:

$$
t_{R n} \operatorname{CL}\left(\frac{\mathrm{h}}{\text { día }}\right)=\left(\frac{n^{\circ} \text { cont.CL } n_{n} * 1,25}{60}\right)
$$

- Tiempo de recogida de contenedores de Carga Trasera en el municipio n:

$$
t_{R n} \mathrm{CT}\left(\frac{\mathrm{h}}{\text { día }}\right)=\left(\frac{n^{\mathrm{o}} \text { cont.CTp } n_{n} * 0,55+n^{\circ} \operatorname{cont}_{\text {C } C T g_{n} * 0,75}}{60}\right)
$$

Para el conjunto de municipios del territorio considerado sería:

- Tiempo de recogida de contenedores de Carga Lateral en el conjunto de municipios:

$$
t_{R} C L\left(\frac{\mathrm{h}}{\text { día }}\right)=\left(\frac{\left(\sum_{n=1}^{n} n^{\mathrm{o}} \operatorname{cont} . C L_{n}\right) * 1,25}{60}\right)
$$

- Tiempo de recogida de contenedores de Carga Trasera en el conjunto de municipios:

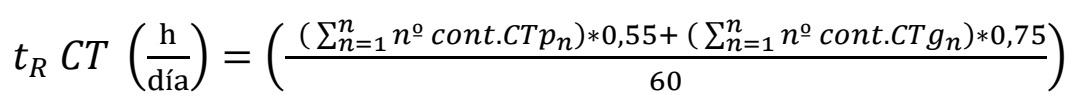

Siendo,

- $t_{R n}$ CL: Tiempo empleado en la recogida de contenedores de Carga Lateral del municipio n (h/día).

- $t_{\mathrm{Rn}} \mathrm{CT}$ : Tiempo empleado en la recogida de contenedores de Carga Trasera del municipio n (h/día).

- $t_{R}$ CL: Tiempo empleado en la recogida de contenedores de Carga Lateral para el conjunto de municipios del territorio considerado (h/día).

- $t_{R}$ CT: Tiempo empleado en la recogida de contenedores de Carga Trasera para el 
conjunto de municipios del territorio considerado (h/día).

- $\mathrm{n}^{\circ}$ cont. CLn : Número de contenedores de Carga Lateral del municipio $\mathrm{n}$.

- $\mathrm{n}^{\mathrm{o}}$ cont. CTp $\mathrm{n}$ : Número de contenedores de Carga Trasera pequeños del municipio n.

- $\mathrm{n}^{\mathrm{o}}$ cont. $\mathrm{CTg}_{\mathrm{n}}$ : Número de contenedores de Carga Trasera grandes del municipio n.

Una vez obtenidos $t_{R} C L$ y $t_{R} C T$, multiplicamos por el consumo de combustible diésel (computado en litros/hora) asociado a cada vehículo en función de su sistema de carga (no se computa el consumo en litros/km ya que el vehículo cuando está levantando los contenedores no se desplaza, pero el motor sí está en funcionamiento) (Fernández, 2012):

- $\quad 8,83 \mathrm{~L} / \mathrm{h}$ para camiones recolectores de Carga Lateral

- $11,38 \mathrm{~L} / \mathrm{h}$ para camiones recolectores de Carga Trasera

De este modo obtenemos el consumo diario (L/día) asociado a la totalidad de levantadas de Carga Lateral o Trasera pertenecientes a un territorio. Para obtener el consumo anual, K (L/año), suponemos que en los dos territorios estudiados la recogida de la fracción orgánica y resto se realiza 6 días en semana y 52 semanas al año, de modo que el consumo anual $\mathrm{K}$ (L/año), correspondiente con la recogida urbana de los dos sistemas de carga (Lateral y Trasera) y para la totalidad de las rutas del territorio considerado es:

$$
\begin{gathered}
\text { Consumo anual }=K\left(\frac{L}{\text { año }}\right)=\left(t_{R} C L\left(\frac{\mathrm{h}}{\text { día }}\right) * 8,83\left(\frac{\mathrm{L}}{\mathrm{h}}\right)+t_{R} C T\left(\frac{\mathrm{h}}{\text { día }}\right) * 11,38\left(\frac{\mathrm{L}}{\mathrm{h}}\right)\right) * \\
6\left(\frac{\text { días }}{\text { semana }}\right) * 52\left(\frac{\text { semanas }}{a \tilde{n} o}\right)
\end{gathered}
$$

Finalmente, para obtener las emisiones derivadas de la recogida $\left(E_{R}\right)$ multiplicamos el consumo anual $\mathrm{K}$ (L/año), por un ratio de emisiones de $\mathrm{KgCO}_{2 \text { eq }}$ por litro de combustible consumido y dividimos entre las toneladas anuales generadas en el territorio considerado $(\mathrm{G}$, en Tn/año). Dicho ratio de emisiones, según el Panel Intergubernamental sobre el Cambio Climático (Intergovernmental Panel on Climate Change, IPCC), es del orden de 74.100 kilogramos por Terajulio de Diésel (Eggleston et al., 2006), lo que equivale aproximadamente a 2,65 kilogramos de $\mathrm{CO}_{2 \text { eq }}$ por litro de Diésel consumido (Moliner et al., 2011; OCCC, 2013).

$$
E_{R}\left(\frac{K g \mathrm{CO}_{2 e q}}{T n}\right)=K\left(\frac{L}{a \tilde{n} o}\right) * 2,65\left(\frac{K g C O_{2 e q}}{L}\right) * \frac{1}{G\left(\frac{T n}{a \tilde{n} o}\right)}
$$

Siendo,

- $\mathrm{E}_{\mathrm{R}}$ : Emisiones derivadas de la recogida de RU en el territorio considerado $\left(\mathrm{KgCO}_{2 \mathrm{eq}} / \mathrm{Tn}\right)$.

- K: Consumo anual de combustible diésel empleado por los camiones recolectores de Carga Lateral o Carga Trasera en la totalidad de levantadas de un territorio (L/año).

- G: Generación anual de RU en el territorio considerado (Tn/año). 


\subsubsection{Emisiones derivadas del transporte, $\left(\mathrm{E}_{\mathrm{T}}\right)$}

Son las emitidas por el camión recolector de RU cuando se encuentra en movimiento, ya sea por carretera urbana $\left(\mathrm{E}_{\mathrm{TR}}\right)$, carretera interurbana $\left(\mathrm{E}_{\mathrm{TI}}\right)$ o autovía $\left(\mathrm{E}_{\mathrm{TA}}\right)$.

$$
E_{T}\left(\frac{K g C O_{2 e q}}{T n}\right)=E_{T U}\left(\frac{K g C O_{2 e q}}{T n}\right)+E_{T I}\left(\frac{K g C O_{2 e q}}{T n}\right)+E_{T A}\left(\frac{K g C O_{2 e q}}{T n}\right)
$$

Siendo,

- $\mathrm{E}_{\mathrm{T}}$ : Emisiones derivadas del transporte de RU en el territorio considerado $\left(\mathrm{KgCO}_{2 \mathrm{eq}} / \mathrm{Tn}\right)$.

- $\mathrm{E}_{\mathrm{TU}}$ : Emisiones derivadas del transporte de RU por carretera urbana en el territorio considerado $\left(\mathrm{KgCO}_{2 \mathrm{eq}} / \mathrm{Tn}\right)$.

- $\mathrm{E}_{\mathrm{TI}}$ : Emisiones derivadas del transporte de RU por carretera interurbana en el territorio considerado $\left(\mathrm{KgCO}_{2 \mathrm{eq}} / \mathrm{Tn}\right)$.

- $\mathrm{E}_{\mathrm{TA}}$ : Emisiones derivadas del transporte de RU por autovía en el territorio considerado $\left(\mathrm{KgCO}_{2 \mathrm{eq}} / \mathrm{Tn}\right)$.

Estos tres sumandos dependen a su vez de los siguientes tiempos:

1. Tiempo de Transporte Urbano, $\mathrm{t}_{\mathrm{TU}}$ ( $\mathrm{h} /$ día): tiempo diario empleado en los desplazamientos del camión recolector entre ubicaciones de contenedores. Este tiempo se calcula para un municipio n como:

$$
t_{T U n}\left(\frac{h}{d i ́ a}\right)=t_{U n}\left(\frac{h}{d^{i} a}\right)-t_{R n} C L\left(\frac{h}{d i ́ a}\right)-t_{R n} C T\left(\frac{h}{d^{\prime} a}\right)
$$

Para el conjunto de municipios del territorio de estudio sería:

$$
t_{T U}\left(\frac{h}{d i ́ a}\right)=\sum_{n=1}^{n} t_{U n}\left(\frac{h}{d i ́ a}\right)-t_{R} C L\left(\frac{h}{d i ́ a}\right)-t_{R} C T\left(\frac{h}{d i ́ a}\right)
$$

Siendo:

- $\mathrm{t}_{\mathrm{TUn}}$ : Tiempo de transporte por carretera urbana en el municipio $\mathrm{n}(\mathrm{h} / \mathrm{día})$.

- $t_{U n}$ : Tiempo urbano en el municipio $\mathrm{n}$ (h/día). Se calcula como:

$$
t_{U n}\left(\frac{h}{\text { día }}\right)=t_{R n}\left(\frac{h}{\text { día }}\right)+t_{T U n}\left(\frac{h}{\text { día }}\right)
$$

- $\quad \mathrm{t}_{\mathrm{TU}}$ : Tiempo de transporte por carretera urbana para el conjunto de municipios del territorio considerado ( $\mathrm{h} /$ día).

- $t_{R n}$ CL: Tiempo empleado en la recogida de contenedores de Carga Lateral del municipio n (h/día).

- $t_{R n}$ CT: Tiempo empleado en la recogida de contenedores de Carga Trasera del municipio n (h/día).

- $t_{\mathrm{Rn}}$ : Tiempo empleado en la recogida de la totalidad de contenedores del municipio $\mathrm{n}$ (h/día). Se calcula como:

$$
t_{R n}\left(\frac{h}{d i ́ a}\right)=t_{R n} C L\left(\frac{h}{d i ́ a}\right)+t_{R n} C T\left(\frac{h}{d i ́ a}\right)
$$


2. Tiempo de Transporte Interurbano, $\mathrm{t}_{\mathrm{TI}}$ (h/día): tiempo diario empleado por el camión recolector cuando va circulando por vías interurbanas (entre municipio y municipio y entre la base de salida y el municipio). Para un municipio n, este tiempo es conocido según datos de las tablas 7, 8 y 12. Para el conjunto de municipios de un territorio se calcularía como:

$$
t_{T I}\left(\frac{h}{d i ́ a}\right)=\sum_{n=1}^{n} t_{T I n}\left(\frac{h}{d i ́ a}\right)
$$

Siendo,

- $\mathrm{t}_{\mathrm{TI}}$ : Tiempo de transporte por carretera interurbana para el conjunto de municipios del territorio considerado (h/día).

- $\mathrm{t}_{\mathrm{TIn}}$ : Tiempo de transporte por carretera interurbana en el municipio $\mathrm{n}(\mathrm{h} / \mathrm{día})$.

3. Tiempo de Transporte por Autovía, $\mathrm{t}_{\mathrm{TA}}$ (h/día): tiempo diario empleado por el camión recolector cuando circula desde el último municipio recogido hasta la planta de tratamiento mecánico biológico (PTMB) o planta de transferencia (PT) de destino final de los RU y la vuelta al municipio o base de salida. Para un municipio n, este tiempo se obtiene de las tablas 7, 8 y 12. Para el conjunto de municipios de un territorio se calcularía como:

$$
t_{T A}\left(\frac{h}{\text { día }^{\prime}}\right)=\sum_{n=1}^{n} t_{T A n}\left(\frac{h}{d i ́ a}\right)
$$

Siendo,

- $\mathrm{t}_{\mathrm{TA}}$ : Tiempo de transporte por autovía para el conjunto de municipios del territorio considerado (h/día).

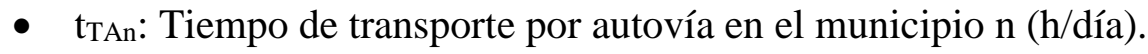

Calculados $\mathrm{t}_{\mathrm{TU}}, \mathrm{t}_{\mathrm{TI}} \mathrm{y} \mathrm{t}_{\mathrm{TA}}$ (h/día) y suponiendo que en los dos territorios estudiados la recogida se lleva a cabo 6 días a la semana y 52 semanas al año, se puede calcular la distancia anual recorrida en cada tipo de vía $\mathrm{D}_{\mathrm{TU}}, \mathrm{D}_{\mathrm{TI}}$ y $\mathrm{D}_{\mathrm{TA}}(\mathrm{Km} / \mathrm{año})$, asignando una velocidad media para los camiones de (Burón et al., 2004):

- Vías urbanas: $14 \mathrm{~km} / \mathrm{h}$

- Vías interurbanas: $50 \mathrm{~km} / \mathrm{h}$

- Autopistas y autovías: $70 \mathrm{~km} / \mathrm{h}$

$$
\begin{aligned}
& D_{T U}\left(\frac{K m}{a \tilde{n} o}\right)=t_{T U}\left(\frac{h}{\text { día }}\right) * 6\left(\frac{\text { días }}{\text { semana }}\right) * 52\left(\frac{\text { semanas }}{a \tilde{n} o}\right) * 14\left(\frac{K m}{h}\right) \\
& D_{T I}\left(\frac{K m}{\text { año }}\right)=t_{T I}\left(\frac{h}{\text { día }}\right) * 6\left(\frac{\text { días }}{\text { semana }}\right) * 52\left(\frac{\text { semanas }}{a \tilde{n} o}\right) * 50\left(\frac{K m}{h}\right) \\
& D_{T A}\left(\frac{K m}{\text { año }}\right)=t_{T A}\left(\frac{h}{\text { día }}\right) * 6\left(\frac{\text { días }}{\text { semana }}\right) * 52\left(\frac{\text { semanas }}{\text { año }}\right) * 70\left(\frac{K m}{h}\right)
\end{aligned}
$$

Siendo,

- $\mathrm{D}_{\mathrm{TU}}$ : Distancia anual recorrida por carretera urbana por los camiones recolectores del territorio considerado (Km/año).

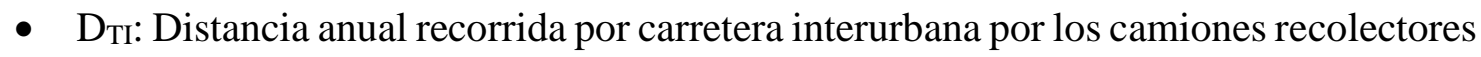


del territorio considerado (Km/año).

- $\mathrm{D}_{\mathrm{TA}}$ : Distancia anual recorrida por autovía por los camiones recolectores del territorio considerado (Km/año).

Para obtener las emisiones derivadas del transporte según el tipo de vía de circulación $\mathrm{E}_{\mathrm{TU}}$, $\mathrm{E}_{\text {TI y }} \mathrm{E}_{\mathrm{TA}}\left(\mathrm{KgCO}_{2 \mathrm{eq}} / \mathrm{Tn}\right)$, multiplicamos la distancia anual asociada a cada tipo de vía $\mathrm{D}_{\mathrm{TU}}$, $\mathrm{D}_{\mathrm{TI}}$ y $\mathrm{D}_{\mathrm{TA}}(\mathrm{Km} / \mathrm{año})$ por un ratio de emisiones de $\mathrm{KgCO}_{2 \text { eq }}$ por $\mathrm{Km}$ recorrido y dividimos entre las toneladas anuales generadas en el territorio considerado ( $\mathrm{G}$, en Tn/año). Dicho ratio de emisiones es, para camiones de tipo diésel rígido (OCCC, 2013):

- Vías urbanas $(1.295,62 \mathrm{gCO} 2 \mathrm{eq} / \mathrm{km})$

- Vías interurbanas $\left(562,33 \mathrm{gCO}_{2 \mathrm{eq}} / \mathrm{km}\right)$

- Autopistas y autovías $\left(518,68 \mathrm{gCO}_{2 \mathrm{eq}} / \mathrm{km}\right)$

$$
\begin{gathered}
E_{T U}\left(\frac{K g C O_{2 e q}}{T n}\right)=D_{T U}\left(\frac{k m}{a \tilde{n} o}\right) * 1.295,62\left(\frac{g C O_{2 e q}}{k m}\right) * \frac{1}{1000}\left(\frac{K g C O_{2 e q}}{g C O_{2 e q}}\right) * \frac{1}{G\left(\frac{T n}{a \tilde{o} o}\right)} \\
E_{T I}\left(\frac{K g C O_{2 e q}}{T n}\right)=D_{T I}\left(\frac{k m}{a \tilde{n} o}\right) * 562,33\left(\frac{g C O_{2 e q}}{k m}\right) * \frac{1}{1000}\left(\frac{K g C O_{2 e q}}{g C O_{2 e q}}\right) * \frac{1}{G\left(\frac{T n}{a \tilde{o} o}\right)} \\
E_{T A}\left(\frac{K g C O_{2 e q}}{T n}\right)=D_{T A}\left(\frac{k m}{a \tilde{o} o}\right) * 518,68\left(\frac{g C O_{2 e q}}{k m}\right) * \frac{1}{1000}\left(\frac{K g C O_{2 e q}}{g C O_{2 e q}}\right) * \frac{1}{G\left(\frac{T n}{a \tilde{n} o}\right)}
\end{gathered}
$$

Siendo,

- $\mathrm{E}_{\mathrm{TU}}$ : Emisiones derivadas del transporte de RU por carretera urbana $\left(\mathrm{KgCO}_{2 \mathrm{eq}} / \mathrm{Tn}\right)$.

- ETI: Emisiones derivadas del transporte de RU por carretera interurbana $\left(\mathrm{KgCO}_{2 \mathrm{eq}} / \mathrm{Tn}\right)$.

- $\mathrm{E}_{\mathrm{TA}}$ : Emisiones derivadas del transporte de $\mathrm{RU}$ por autovía $\left(\mathrm{KgCO}_{2 \mathrm{eq}} / \mathrm{Tn}\right)$.

- G: Generación anual de RU en el territorio considerado (Tn/año).

\section{RESULTADOS Y DISCUSIÓN}

Los resultados de este estudio para la obtención del beneficio económico y ambiental en los dos territorios estudiados se muestran a continuación.

\subsection{Análisis económico}

Los resultados del análisis económico para el CVSE (Granada) y para el CVB (Alicante) son los siguientes.

\subsubsection{Territorio 1. Consorcio para el Desarrollo de la Vega Sierra Elvira, CVSE} (Granada)

\begin{tabular}{|c|c|c|}
\hline Parámetro & Cálculo & Resultado \\
\hline$\Sigma \mathrm{I}_{\mathrm{n}}(€ /$ año $)$ & Tabla 4 & $2.437 .324,90$ \\
\hline$\Sigma \mathrm{G}_{\mathrm{n}}(\mathrm{Tn} / \mathrm{año})$ & Tabla 4 & $31.634,49$ \\
\hline $\mathrm{Ci}(€ / \mathrm{Tn})$ & Ecuación 3 & 77,05 \\
\hline
\end{tabular}

Tabla 13 - Costes del servicio en el territorio 1. Escenario individualizado. 


\begin{tabular}{|c|c|c|}
\hline Parámetro & Cálculo & Resultado \\
\hline Cm $(€ /$ Tn $)$ & $\begin{array}{c}\text { Conocido Oferta } \\
\text { Adjudicada }\end{array}$ & 63,90 \\
\hline
\end{tabular}

Tabla 14 - Costes del servicio en el territorio 1. Escenario mancomunado.

\begin{tabular}{|c|c|c|}
\hline Parámetro & Cálculo & Resultado \\
\hline $\mathrm{Ci}(€ / \mathrm{Tn})$ & Ecuación 3 & 77,05 \\
\hline $\mathrm{Cm}(€ / \mathrm{Tn})$ & $\begin{array}{c}\text { Conocido Oferta } \\
\text { Adjudicada }\end{array}$ & 63,90 \\
\hline $\begin{array}{c}\text { Beneficio Económico } \\
(€ / \mathrm{Tn})\end{array}$ & Ecuación 1 & $13,15(\downarrow 17 \%)$ \\
\hline
\end{tabular}

Tabla 15 - Beneficio económico en el territorio 1.

3.1.2. Territorio 2. Consorcio de la Vega Baja, CVB (Alicante)

\begin{tabular}{|c|c|c|}
\hline Parámetro & Cálculo & Resultado \\
\hline$\Sigma \mathrm{Ii}_{\mathrm{n}}(€ / \mathrm{año})$ & Tabla 10 & $1.971 .475,05$ \\
\hline$\Sigma \mathrm{G}_{\mathrm{n}}(\mathrm{Tn} / \mathrm{año})$ & Tabla 10 & $34.981,42$ \\
\hline $\mathrm{Ci}(€ / \mathrm{Tn})$ & Ecuación 3 & 56,36 \\
\hline
\end{tabular}

Tabla 16 - Costes del servicio en el territorio 2. Escenario individualizado.

\begin{tabular}{|c|c|c|}
\hline Parámetro & Cálculo & Resultado \\
\hline Cm $(€ / T n)$ & Estimación & 51,19 \\
\hline
\end{tabular}

Tabla 17 - Costes del servicio en el territorio 2. Escenario mancomunado (estimación).

\begin{tabular}{|c|c|c|}
\hline Parámetro & Cálculo & Resultado \\
\hline $\mathrm{Ci}(€ / \mathrm{Tn})$ & Ecuación 3 & 56,36 \\
\hline $\mathrm{Cm}(€ / \mathrm{Tn})$ & Estimación & 51,19 \\
\hline $\begin{array}{c}\text { Beneficio Económico } \\
(€ / \mathrm{Tn})\end{array}$ & Ecuación 1 & $5,17(\downarrow 9 \%)$ \\
\hline
\end{tabular}

Tabla 18 - Beneficio económico en el territorio 2 (estimación).

\subsection{Análisis ambiental}

Los resultados del análisis ambiental para el CVSE (Granada) y para el CVB (Alicante) son los siguientes.

3.2.1. Territorio 1. Consorcio para el Desarrollo de la Vega Sierra Elvira, CVSE (Granada)

\begin{tabular}{|c|c|c|}
\hline Parámetro & Cálculo & Resultado \\
\hline$\Sigma \mathrm{N}^{\mathrm{o}}$ cont. $\mathrm{CL}$ & Tabla 5 & 181 \\
\hline$\Sigma \mathrm{N}^{\mathrm{o}}$ cont. $\mathrm{CTp}$ & Tabla 5 & 267 \\
\hline$\Sigma \mathrm{N}^{\mathrm{o}}$ cont. $\mathrm{CTg}$ & Tabla 5 & 1.405 \\
\hline G (Tn/año) & Tabla 4 & $31.634,49$ \\
\hline $\mathrm{t}_{\mathrm{R}} \mathrm{CL}$ (h/día) & Ecuación 13 & $3: 46: 15$ \\
\hline $\mathrm{t}_{\mathrm{R}} \mathrm{CT}$ (h/día) & Ecuación 14 & $20: 00: 36$ \\
\hline K (L/año) & Ecuación 15 & $81.435,20$ \\
\hline $\mathrm{E}_{\mathrm{Ri}}\left(\mathrm{KgCO}_{2 \mathrm{eq}} / \mathrm{Tn}\right)$ & Ecuación 16 & 6,82 \\
\hline$\Sigma$ tu $_{\mathrm{U}}$ (h/día) & Tabla 7 & $43: 40: 00$ \\
\hline $\mathrm{t}_{\mathrm{TU}}$ (h/día) & Ecuación 19 & 19:53:09 \\
\hline $\mathrm{t}_{\mathrm{TI}}(\mathrm{h} / \mathrm{dí} \mathrm{a})$ & Tabla 7 & $4: 17: 16$ \\
\hline
\end{tabular}




\begin{tabular}{|c|c|c|}
\hline $\mathrm{t}_{\mathrm{TA}}(\mathrm{h} /$ día $)$ & Tabla 7 & $11: 45: 07$ \\
\hline $\mathrm{D}_{\mathrm{TU}}(\mathrm{Km} / \mathrm{año})$ & Ecuación 24 & $86.861,32$ \\
\hline $\mathrm{D}_{\mathrm{TI}}(\mathrm{Km} / \mathrm{año})$ & Ecuación 25 & $66.889,33$ \\
\hline $\mathrm{D}_{\mathrm{TA}}(\mathrm{Km} / \mathrm{año})$ & Ecuación 26 & $256.662,47$ \\
\hline $\mathrm{E}_{\mathrm{TUi}}\left(\mathrm{KgCO}_{2 \mathrm{eq}} / \mathrm{Tn}\right)$ & Ecuación 27 & 3,56 \\
\hline $\mathrm{E}_{\mathrm{TTi}}\left(\mathrm{KgCO}_{2 \mathrm{eq}} / \mathrm{Tn}\right)$ & Ecuación 28 & 1,19 \\
\hline $\mathrm{E}_{\mathrm{TAi}}\left(\mathrm{KgCO}_{2 \mathrm{eq}} / \mathrm{Tn}\right)$ & Ecuación 29 & 8,21 \\
\hline $\mathrm{E}_{\mathrm{Ti}}\left(\mathrm{KgCO}_{2 \mathrm{eq}} / \mathrm{Tn}\right)$ & Ecuación 17 & 15,78 \\
\hline $\mathrm{E}_{\mathrm{i}}\left(\mathrm{KgCO}_{2 \mathrm{eq}} / \mathrm{Tn}\right)$ & Ecuación 7 & 15 \\
\hline
\end{tabular}

Tabla 19 - Emisiones derivadas de la recogida y transporte en el territorio 1. Escenario individualizado.

\begin{tabular}{|c|c|c|}
\hline Parámetro & Cálculo & Resultado \\
\hline$\Sigma \mathrm{N}^{\circ}$ cont. $\mathrm{CL}$ & Tabla 6 & 655 \\
\hline$\Sigma \mathrm{N}^{\mathrm{o}}$ cont. $\mathrm{CTp}$ & Tabla 6 & 87 \\
\hline$\Sigma \mathrm{N}^{\mathrm{o}}$ cont. $\mathrm{CTg}$ & Tabla 6 & 423 \\
\hline G (Tn/año) & Tabla 4 & $31.634,49$ \\
\hline $\mathrm{t}_{\mathrm{R}} \mathrm{CL}(\mathrm{h} / \mathrm{día})$ & Ecuación 13 & $13: 38: 45$ \\
\hline $\mathrm{t}_{\mathrm{R}} \mathrm{CT}$ (h/día) & Ecuación 14 & $6: 05: 06$ \\
\hline K (L/año) & Ecuación 15 & $59.198,88$ \\
\hline $\mathrm{E}_{\mathrm{Rm}}\left(\mathrm{KgCO}_{2 \mathrm{eq}} / \mathrm{Tn}\right)$ & Ecuación 16 & 4,96 \\
\hline$\Sigma \mathrm{t}_{\mathrm{U}}(\mathrm{h} /$ día $)$ & Tabla 8 & $39: 13: 36$ \\
\hline tTU (h/día) & Ecuación 19 & $19: 29: 45$ \\
\hline $\mathrm{t}_{\mathrm{TI}}$ (h/día) & Tabla 8 & $6: 15: 27$ \\
\hline $\mathrm{t}_{\mathrm{TA}}(\mathrm{h} /$ día $)$ & Tabla 8 & $10: 50: 41$ \\
\hline $\mathrm{D}_{\mathrm{TU}}(\mathrm{Km} / \mathrm{año})$ & Ecuación 24 & $85.157,80$ \\
\hline $\mathrm{D}_{\mathrm{TI}}(\mathrm{Km} / \mathrm{año})$ & Ecuación 25 & $97.617,00$ \\
\hline $\mathrm{D}_{\mathrm{TA}}(\mathrm{Km} / \mathrm{año})$ & Ecuación 26 & $236.848,73$ \\
\hline $\mathrm{E}_{\mathrm{TUm}}\left(\mathrm{KgCO}_{2 \mathrm{eq}} / \mathrm{Tn}\right)$ & Ecuación 27 & 3,49 \\
\hline $\mathrm{E}_{\mathrm{TIm}}\left(\mathrm{KgCO}_{2 \mathrm{eq}} / \mathrm{Tn}\right)$ & Ecuación 28 & 1,74 \\
\hline $\mathrm{E}_{\mathrm{TAm}}\left(\mathrm{KgCO}_{2 \mathrm{eq}} / \mathrm{Tn}\right)$ & Ecuación 29 & 3,88 \\
\hline $\mathrm{E}_{\mathrm{Tm}}\left(\mathrm{KgCO}_{2 \mathrm{eq}} / \mathrm{Tn}\right)$ & Ecuación 17 & 9,11 \\
\hline $\mathrm{E}_{\mathrm{m}}\left(\mathrm{KgCO}_{2 \mathrm{eq}} / \mathrm{Tn}\right)$ & Ecuación 8 & 14,07 \\
\hline
\end{tabular}

Tabla 20 - Emisiones derivadas de la recogida y transporte en el territorio 1. Escenario mancomunado.

\begin{tabular}{|c|c|c|}
\hline Parámetro & Cálculo & Resultado \\
\hline $\mathrm{E}_{\mathrm{i}}\left(\mathrm{KgCO}_{2 \mathrm{eq}} / \mathrm{Tn}\right)$ & Ecuación 7 & 15,78 \\
\hline $\mathrm{E}_{\mathrm{m}}\left(\mathrm{KgCO}_{2 \mathrm{eq}} / \mathrm{Tn}\right)$ & Ecuación 8 & 14,07 \\
\hline $\begin{array}{c}\text { Beneficio Ambiental } \\
\left(\mathrm{KgCO}_{2 \mathrm{eq}} / \mathrm{Tn}\right)\end{array}$ & Ecuación 6 & $1,71(\downarrow 11 \%)$ \\
\hline
\end{tabular}

Tabla 21 - Beneficio ambiental en el territorio 1. 


\subsubsection{Territorio 2. Consorcio de la Vega Baja, CVB (Alicante)}

\begin{tabular}{|c|c|c|}
\hline Parámetro & Cálculo & Resultado \\
\hline$\Sigma \mathrm{N}^{\mathrm{o}}$ cont. $\mathrm{CL}$ & Tabla 11 & 0 \\
\hline$\Sigma \mathrm{N}^{\mathrm{o}}$ cont. $\mathrm{CTp}$ & Tabla 11 & 109 \\
\hline$\Sigma \mathrm{N}^{\mathrm{o}}$ cont. CTg & Tabla 11 & 2.593 \\
\hline G (Tn/año) & Tabla 10 & $34.981,42$ \\
\hline$t_{R} C L(h /$ día $)$ & Ecuación 13 & 0 \\
\hline $\mathrm{t}_{\mathrm{R}} \mathrm{CT}$ (h/día) & Ecuación 14 & $33: 24: 42$ \\
\hline K (L/año) & Ecuación 15 & $118.630,13$ \\
\hline $\mathrm{E}_{\mathrm{Ri}}\left(\mathrm{KgCO}_{2 \mathrm{eq}} / \mathrm{Tn}\right)$ & Ecuación 16 & 8,99 \\
\hline$\Sigma \mathrm{t}_{\mathrm{U}}(\mathrm{h} /$ día $)$ & Tabla 12 & $51: 54: 39$ \\
\hline tTU (h/día) & Ecuación 19 & $18: 29: 57$ \\
\hline $\mathrm{t}_{\mathrm{TI}}(\mathrm{h} / \mathrm{día})$ & Tabla 12 & $0: 28: 57$ \\
\hline tTA $(\mathrm{h} /$ día $)$ & Tabla 12 & 21:20:03 \\
\hline $\mathrm{D}_{\mathrm{TU}}(\mathrm{Km} / \mathrm{año})$ & Ecuación 24 & $80.804,36$ \\
\hline $\mathrm{D}_{\mathrm{TI}}(\mathrm{Km} / \mathrm{año})$ & Ecuación 25 & $7.525,44$ \\
\hline $\mathrm{D}_{\mathrm{TA}}(\mathrm{Km} / \mathrm{a} \tilde{n} \mathrm{O})$ & Ecuación 26 & $465.940,80$ \\
\hline $\mathrm{E}_{\mathrm{TUi}}\left(\mathrm{KgCO}_{2 \mathrm{eq}} / \mathrm{Tn}\right)$ & Ecuación 27 & 2,99 \\
\hline $\mathrm{E}_{\mathrm{TIi}}\left(\mathrm{KgCO}_{2 \mathrm{eq}} / \mathrm{Tn}\right)$ & Ecuación 28 & 0,12 \\
\hline $\mathrm{E}_{\mathrm{TAi}}\left(\mathrm{KgCO}_{2 \mathrm{eq}} / \mathrm{Tn}\right)$ & Ecuación 29 & 6,91 \\
\hline $\mathrm{E}_{\mathrm{Ti}}\left(\mathrm{KgCO}_{2 \mathrm{eq}} / \mathrm{Tn}\right)$ & Ecuación 17 & 10,02 \\
\hline $\mathrm{E}_{\mathrm{i}}\left(\mathrm{KgCO}_{2 \mathrm{eq}} / \mathrm{Tn}\right)$ & Ecuación 7 & 19,01 \\
\hline
\end{tabular}

Tabla 22 - Emisiones derivadas de la recogida y transporte en el territorio 2. Escenario individualizado.

\begin{tabular}{|c|c|c|}
\hline Parámetro & Cálculo & Resultado \\
\hline $\mathrm{G}(\mathrm{Tn} / \mathrm{año})$ & Tabla 10 & $34.981,42$ \\
\hline $\mathrm{E}_{\mathrm{Rm}}\left(\mathrm{KgCO}_{2 \mathrm{eq}} / \mathrm{Tn}\right)$ & Estimación & 7,28 \\
\hline $\mathrm{E}_{\mathrm{TUm}}\left(\mathrm{KgCO}_{2 \mathrm{eq}} / \mathrm{Tn}\right)$ & Estimación & 2,85 \\
\hline $\mathrm{E}_{\mathrm{TIm}}\left(\mathrm{KgCO}_{2 \mathrm{eq}} / \mathrm{Tn}\right)$ & Estimación & 0,22 \\
\hline $\mathrm{E}_{\mathrm{TAm}}\left(\mathrm{KgCO}_{2 \mathrm{eq}} / \mathrm{Tn}\right)$ & Estimación & 6,57 \\
\hline $\mathrm{E}_{\mathrm{Tm}}\left(\mathrm{KgCO}_{2 \mathrm{eq}} / \mathrm{Tn}\right)$ & Estimación & 9,64 \\
\hline $\mathrm{E}_{\mathrm{m}}\left(\mathrm{KgCO}_{2 \mathrm{eq}} / \mathrm{Tn}\right)$ & Estimación & 16,92 \\
\hline
\end{tabular}

Tabla 23 - Emisiones derivadas de la recogida y transporte en el territorio 2. Escenario mancomunado (estimación).

\begin{tabular}{|c|c|c|}
\hline Parámetro & Cálculo & Resultado \\
\hline $\mathrm{E}_{\mathrm{i}}\left(\mathrm{KgCO}_{2 \mathrm{eq}} / \mathrm{Tn}\right)$ & Ecuación 7 & 19,01 \\
\hline $\mathrm{E}_{\mathrm{m}}\left(\mathrm{KgCO}_{2 \mathrm{eq}} / \mathrm{Tn}\right)$ & Estimación & 16,92 \\
\hline $\begin{array}{c}\text { Beneficio Ambiental } \\
\left(\mathrm{KgCO}_{2 \mathrm{eq}} / \mathrm{Tn}\right)\end{array}$ & Ecuación 6 & $2,09(\downarrow 11 \%)$ \\
\hline
\end{tabular}

Tabla 24 - Beneficio ambiental en el territorio 2 (estimación). 


\subsection{Discusión resultados}

Los resultados del análisis económico obtenidos para el territorio 1 (CVSE, Granada) demuestran que la mancomunación del servicio de recogida y transporte de RU, así como la implantación de nuevas tecnologías, han permitido alcanzar un beneficio económico del $17 \%$, pasando de ser el coste del servicio por tonelada de RU generada de 77,05€/Tn -en el escenario individualizado-, a 63,90 €/Tn -en el escenario mancomunado-, ambos costes con IVA incluido.

En base a estos resultados, se estima que de aplicarse estas medidas en el territorio 2 (CVB, Alicante), el beneficio económico podría alcanzar como mínimo el 9\%, pasando este territorio de un coste por tonelada de RU generada de 56,36€/Tn, a 51,19 €/Tn -en el escenario mancomunado-, ambos costes con IVA incluido.

El beneficio económico derivado de la mancomunación de los servicios de recogida y transporte de RU era de esperar en base al estudio de Lombrano (2009) y además se ve optimizado por la implantación de nuevas tecnologías, como ya determinó Sánchez (2015).

Por otro lado, los resultados del análisis ambiental calculados para el territorio 1 (CVSE, Granada) han demostrado que la mancomunación del servicio de recogida y transporte de RU, así como la implantación de nuevas tecnologías, permiten obtener un beneficio ambiental de hasta el $11 \%$, pasando de unas emisiones de $\mathrm{CO}_{2 \text { eq }}$ por tonelada de RU generada de $15,78 \mathrm{KgCO}_{2 \mathrm{eq}} / \mathrm{Tn}$-en el escenario individualizado-, a $14,07 \mathrm{KgCO}_{2 \mathrm{eq}} / \mathrm{Tn}$-en el escenario mancomunado-. Así mismo, se demuestra con este análisis que las emisiones asociadas a la recogida de RU disminuyen si se pasa de un sistema de contenerización de carga trasera a carga lateral (ya que esto implica un menor número de contenedores a recoger, por un aumento de volumen del recipiente en Carga Lateral), ocurre lo mismo con las emisiones asociadas al transporte urbano. Las emisiones asociadas al transporte por autovía se reducen en el escenario mancomunado (ya que hay un menor número de rutas) pero sin embargo, las emisiones asociadas al transporte interurbano aumentan respecto al escenario individualizado (por implicar la mancomunación la agrupación de varios municipios y pedanías en una misma ruta).

Estos resultados han permitido estimar que las emisiones actuales del escenario individualizado del territorio 2 (CVB, Alicante), cuantificadas en $19,01 \mathrm{KgCO}_{2 \mathrm{eq}} / \mathrm{Tn}$, se reduzcan hasta en un $11 \%$ en el escenario mancomunado, por lo que pasarían a ser 16,90 $\mathrm{KgCO}_{2 \text { eq }} / \mathrm{Tn}$. Para ello, se ha considerado que, al igual que ocurre en el CVSE (Granada), las emisiones asociadas a la recogida, transporte urbano y transporte por autovía disminuirán en el escenario mancomunado, mientras que las emisiones asociadas al transporte interurbano aumentarán respecto al escenario actual individualizado.

El beneficio ambiental derivado de la mancomunación de los servicios de recogida y transporte de RU era previsible en base a los resultados del estudio de Moliner (2011). Por otro lado, los resultados que se han obtenido de emisiones de $\mathrm{CO}_{2 \text { eq }}$ asociadas a la recogida y transporte de RU (fracción orgánica y resto), van en consonancia con los datos recogidos por la Generalitat de Catalunya (2016), en su guía para el "Càlcul de les emissions de GEH derivades de la gestió dels residus municipals".

\section{CONCLUSIONES}

Los resultados del estudio demuestran que la mancomunación y la implantación de nuevas tecnologías en el servicio de recogida y transporte de RU permiten obtener un beneficio económico y ambiental respecto a situaciones en las que se realizan estas tareas de forma individualizada.

En concreto se ha demostrado que para el territorio 1 de estudio (CVSE, Granada) el 
beneficio económico ha supuesto un ahorro del 17\% de los costes y una reducción del 11\% de emisiones de gases de efecto invernadero (GEI).

En base a estos resultados, se ha estimado que el beneficio económico para el territorio 2 de estudio (CVB, Alicante) será mínimo del 9\% y la reducción de emisiones de gases de efecto invernadero (GEI) puede llegar hasta el $11 \%$.

Se demuestra con este estudio que las recogidas mancomunadas, especialmente para municipios pequeños (que necesiten de economías de escala para optimizar los costes del servicio), son económica y ambientalmente favorables.

La mancomunación de la recogida de RU en municipios de tamaño mediano y pequeño y la implementación de nuevas tecnologías aplicadas a los equipos de recogida, supone una reducción de costes de gestión de los RU para los ciudadanos a la vez que se reducen emisiones de GEI a la atmósfera.

\section{REFERENCIAS}

ABENGOA.

(2007).

http://www.abengoa.es/htmlsites/boletines/es/diciembre2007ext/gestion.htm

Último acceso: 14/05/2016

BOE-A-1985-5392, número 80, de 03/04/1985. Ley 7/1985, de 2 de abril, Reguladora de las Bases del Régimen Local.

BOE-A-2011-13046, número 181, de 29/07/2011. Ley 22/2011, de 28 de julios, de residuos y suelos contaminados.

BURÓN, J.M., LÓPEZ, J.M., APARICIO, F., MARTÍN, M.A. \& GARCÍA, A. (2004). Estimation of road transport emissions in Spain from 1988 to 1999 using COPERT III program. Atmospheric Environment, 38, pp. 715-724.

CASAS, J., TORRAS, A., GARRIGA, E. \& MARTELL, M. (2005). Gestión de los residuos sólidos urbanos. Los residuos municipales y su gestión. Fundación politécnica de Catalunya. Universidad Politécnica de Catalunya. Editorial Metrópolis, Barcelona (España).

CALVO, A., FEMENIAS, L., FONTOIRA, J.M., FORCADA, R.M., GARCÍA, A., GONZÁLEZ, J.A., MARTÍNEZ, A.J., RIBOT, F., RODRÍGUEZ, A. \& RUFES, E. (1997). La Enciclopedia del Medio Ambiente Urbano. Volumen 7, Recogida de Residuos. Editorial Cerro Alto, Sevilla (España).

EGGLESTON, H.S., BUENDIA, L., MIWA, K., NGARA, T. \& TANABE, K. (2006). Guidelines for National Greenhouse Gas Inventories. IPCC, Intergovernmental Panel on Climate Change. Japón.

EUROSTAT. (2016). http://ec.europa.eu/eurostat/web/products-datasets/-/road_go_na_tgtt 
Último acceso: 19/05/2016

FERNÁNDEZ, B. (2012). Anàlisi de la producció de residus i eficiència en la seva gestió. Escola Tècnica Superior d'Enginyeria Industrial de Barcelona - Enginyeria de Materials. Universitat Politècnica de Catalunya.

GENERALITAT DE CATALUNYA, Departament de Territori i Sostenibilitat, Secretaria de Medi Ambient i Sostenibilitat. OCCC, Oficina Catalana del CANVI Climàtic. (2016). Càlcul de les emissions de GEH derivades de la gestió dels residus municipals. Metodologia per a organitzacions.

HONTORIA, E. y ZAMORANO, M. (2000). Fundamentos del manejo de los residuos urbanos. Madrid: Colegio de Ingenieros de Caminos, Canales y Puertos.

IHOBE, Sociedad Pública de Gestión Ambiental. (2013). Metodologías para el cálculo de emisiones de gases de efecto invernadero. Departamento de Medio Ambiente y Política Territorial. Gobierno Vasco.

INE, Instituto Nacional de Estadística. http://www.ine.es/

Último acceso: 18/05/2016

LOMBRANO, A. (2009). Cost efficiency in the management of solid urban waste. Resources, Conservation and Recycling, 53, pp. 601-611.

MAGRAMA, Ministerio de Agricultura, Alimentación y Medio Ambiente. (2013). Gestión de biorresiduos de competencia municipal. Guía para la implantación de la recogida separada y tratamiento de la fracción orgánica. Madrid.

MOLINER, E., MUÑOZ, C., GARRAÍN, D. \& VIDAL, R. (2011). La huella ecológica del transporte de residuos en la provincia de Castellón. XV Congreso Internacional de Ingeniería de Proyectos, Huesca (España).

OCCC, Oficina Catalana del Canvi Climàtic. (2013). Guía práctica para el cálculo de emisiones de gases de efecto invernadero (GEI).

PRIETO, F., ALFONSO, C., GUADILLA, S., SANTAMARTA, J. \& AVELLANER, J. (2014). Sostenibilidad en España 2014. SOS 2014. Observatorio de la sostenibilidad.

RESUR GRANADA, Consorcio Provincial de Residuos Sólidos Urbanos. (2012a). http://www.resurgranada.es/datos_recogida.php

Último acceso: 20/05/2016 
RESUR GRANADA, Consorcio Provincial de Residuos Sólidos Urbanos. (2012b). http://www.resurgranada.es/plantas transferencia.php

Último acceso: 24/05/2016

SÁNCHEZ, J. (2015). Instrumentos de gestión para la optimización del servicio. Los municipios y la gestión de los residuos. Fundació fórum ambiental. Diputación de Barcelona.

$\begin{array}{lllll}\text { TRANSRSU, } & \text { Transportes } & \text { de } & \text { RSU. }\end{array}$

http://www.transrsu.com/servicios/transporte.html

Último acceso: 24/05/2016

ZAMORANO, M., FERNÁNDEZ-GONZÁLEZ, J. M., GARCÍA-MARTÍNEZ, F. J. \& GARCÍA-MARAVER, A. (2015). Guía sobre competencias locales en materia de gestión de residuos. Granada: Diputación de Granada. 